\title{
INERTIAL MANIFOLDS OF INCOMPRESSIBLE, NONLINEAR BIPOLAR VISCOUS FLUIDS
}

\author{
BY
}

\author{
FREDERICK BLOOM AND WENGE HAO
}

Department of Mathematical Sciences, Northern Illinois University, DeKalb, IL

\begin{abstract}
The existence of an inertial manifold is established for the nonlinear system of equations describing the motion of a bipolar incompressible viscous fluid. In this paper we consider only the case of a spatially periodic velocity field. Existence of an inertial manifold for the model complements earlier work on the existence of compact global attractors for bipolar viscous fluids and serves to further highlight the differences between the bipolar model and the usual model based on the linear Stokes constitutive relation.
\end{abstract}

1. Introduction: Bipolar viscous fluids. The theory of multipolar materials is due to Green and Rivlin [1], [2], who considered the constitutive equations for an elastic, nonviscous material; a model for a bipolar fluid may be found in the paper of Bleustein and Green [3]. Nečas and Šilhavý [4] developed a thermodynamic theory of constitutive equations for multipolar viscous fluids within the framework of the theory of Green and Rivlin [1], [2]; the general constitutive theory developed in [4] is consistent with the principle of material-frame indifference and the second law of thermodynamics as expressed by the Clausius-Duhem inequality. In [5], Bellout, Bloom, and Nečas expanded upon some of the consequences of the multipolar fluid model with particular emphasis on the nonlinear, isothermal, incompressible bipolar case.

The Navier-Stokes model of fluid flow is based upon the Stokes hypothesis, which restricts the relation between the stress tensor and the velocity. By relaxing the constraints of the Stokes hypothesis, the mathematical theory of multipolar viscous fluids generalizes the usual Stokes model in three important respects: it allows for nonlinear constitutive relations between the viscous part of the stress tensor and velocity gradients, it allows for a dependence of the viscous stress on velocity gradients of order two or higher, and it introduces constitutive relations for higher-order stress tensors (moments of stress), which must be present in the balance of energy equation as soon as higher-order velocity gradients are admitted into the theory.

Received December 6, 1993.

1991 Mathematics Subject Classification. Primary 76D, 35K.

Key words and phrases. Bipolar fluid, viscosity, global attractor, inertial manifold.

This research was supported, in part, by ONR Grant N00014-91-J-1002. 
The simplest expression for the viscous stress $\tau^{v}$, which is consistent with the primitive conceptual idea of a viscous fluid, i.e., that $\tau^{\prime \prime}$ be zero when there is no relative motion between neighboring portions of the fluid, is one of the form (see, e.g., Shinbrot [6])

$$
\left\{\begin{array}{l}
\boldsymbol{\tau}^{v}=\mathbf{f}(\nabla \mathbf{v}, \nabla \nabla \mathbf{v}, \ldots) \\
\mathbf{f}(\mathbf{0}, \mathbf{0}, \ldots, \mathbf{0})=\mathbf{0}
\end{array}\right.
$$

where $\mathbf{v}$ is the velocity vector. The Stokes hypothesis consists of the simplifying assumption that, in (1.1), $\mathbf{f}$ depends linearly on the first velocity gradient $\nabla \mathbf{v}$ and is independent of all higher-order velocity gradients. The possible utility of considering more general relations, which allow for a nonlinear velocity-dependent viscosity, has been clearly indicated in Ruelle [7] and various theories of viscous fluid response which allow for nonlinearity in the constitutive theory, as well as the presence of higher-order velocity gradients, have been considered, e.g., in Ladyzhenskaya [8], [9], Kaniel [10], and Du and Gunzburger [11]; perturbations of the Navier-Stokes equations which incorporate higher-order velocity gradients may be found, e.g., in the papers of Lions [12] and Ou and Sritharan [13], [14], and the references cited therein, as well as in the book [15] by Temam.

The constitutive relations for isothermal, nonlinear, incompressible bipolar viscous fluid which were introduced in [5] have the form

$$
\begin{gathered}
\tau_{i j}=-p \delta_{i j}+2 \mu_{0}\left(\epsilon+|\mathbf{e}|^{2}\right)^{-\alpha / 2} e_{i j}-2 \mu_{1} \Delta e_{i j} \\
\tau_{i j k}=2 \mu_{1} \frac{\partial e_{i j}}{\partial x_{k}}
\end{gathered}
$$

with the $\tau_{i j}$ being the (components of the) stress tensor, $\tau_{i j k}$ the first multipolar stress tensor, and $p$ the pressure. In (1.2), (1.3) the $e_{i j}$ are the components of the rate of deformation tensor, i.e.

$$
e_{i j}=\frac{1}{2}\left(\frac{\partial u_{i}}{\partial x_{j}}+\frac{\partial u_{j}}{\partial x_{i}}\right)
$$

while $\epsilon, \mu_{0}, \mu_{1}>0$ and $\alpha, 0 \leq \alpha<1$, are constitutive parameters. Besides the presence of the multipolar stress tensor $\tau_{i j k}$ (which affects only higher-order boundary conditions), and the higher-order velocity gradients, the theory also involves a nonlinear viscosity

$$
\mu=\mu_{0}\left(\epsilon+|\mathbf{e}|^{2}\right)^{-\alpha / 2}
$$

in which $\mu_{0}$ has the form $\mu_{0}=\bar{\mu}_{0} \bar{\epsilon}^{\alpha / 2}$ with $\bar{\mu}_{0}$ the usual Stokes viscosity while $\bar{\epsilon}$ has the same physical dimensions as $\epsilon$, i.e., $t^{-2}$; thus

$$
\mu=\bar{\mu}_{0}\left(\bar{\epsilon} / \epsilon+|\mathbf{e}|^{2}\right)^{\alpha / 2}=\bar{\mu}_{0}\left(\tilde{\epsilon}_{1}+\tilde{\epsilon}_{2}|\mathbf{e}|^{2}\right)^{-\alpha / 2},
$$

$\tilde{\epsilon}_{1}=\epsilon / \bar{\epsilon}$ and $\tilde{\epsilon}_{2}=1 / \bar{\epsilon}$. The constitutive relation (1.2) for the lower-order stress in the bipolar model thus bears the same relation to the usual Stokes model that, e.g., the constitutive relation

$$
\mathbf{D}=\left(\epsilon_{1}+\epsilon_{2}|\mathbf{E}|^{2}\right) \mathbf{E} \quad\left(\epsilon_{1}, \epsilon_{2}>0\right)
$$


which is commonly employed [16] in nonlinear optics (D the electric displacement field, $\mathbf{E}$ the electric field), bears to the usual relation for a linear dielectric medium, i.e.,

$$
\mathbf{D}=\epsilon \mathbf{E} \quad(\epsilon>0)
$$

With $\mu_{0}$ having the form indicated above, viz., $\mu_{0}=\bar{\mu}_{0} \bar{\epsilon}^{\alpha / 2}$, the theory represented by (1.2), (1.3) reduces, in fact, precisely to that given by the Stokes model when $\alpha=\mu_{1}=0$.

The constitutive relations (1.2), (1.3), and the condition of incompressibility, produce for the nonlinear bipolar model of a viscous fluid the following system of nonlinear partial differential equations:

$$
\begin{gathered}
\rho \frac{\partial \mathbf{v}}{\partial t}+\mathbf{v} \cdot \nabla \mathbf{v}=-\nabla p+\nabla \cdot(2 \mu \mathbf{e})-2 \mu_{1} \nabla \cdot(\Delta \mathbf{e})+\rho \mathbf{f} \\
\nabla \cdot \mathbf{v}=0
\end{gathered}
$$

where $\rho$ is the constant density, $\mathbf{f}$ is the external body force vector, and $\mu(|\mathbf{e}|)$ is given by (1.5). The system of equations (1.8), (1.9) holds in some domain $\Omega \times[0, T), \Omega \subseteq R^{n}$, $n=1,2,3, T>0$, and is subject to initial conditions of the form

$$
\mathbf{v}(\mathbf{x}, 0)=\mathbf{v}_{0}(\mathbf{x}), \quad \mathbf{x} \in \Omega
$$

When $\Omega \subseteq R^{n}$ is a bounded domain (1.8)-(1.10) is supplemented by the boundary conditions

$$
\mathbf{v}=\mathbf{0}, \quad \tau_{i j k} \nu_{j} \nu_{k}=0, \quad i=1,2,3, \quad \text { on } \partial \Omega \times[0, T) .
$$

The first set of conditions in (1.11) represents the usual no-slip condition associated with a viscous fluid, while the second set expresses the fact that the first moments of the traction vanish on $\partial \Omega$; it is a direct consequence of the principle of virtual work (e.g., Toupin [16]). In (1.11), $\nu$ represents the exterior unit normal to $\partial \Omega$.

If $\Omega=[0, L]^{n}, L>0$, then we are looking at spatially periodic solutions of (1.8)-(1.10); if $\left\{\mathbf{e}_{1}, \mathbf{e}_{2}, \ldots, \mathbf{e}_{n}\right\}$ is the natural basis of $\mathbb{R}^{n}$ then the spatial periodicity conditions are

$$
\begin{gathered}
v_{i}(\mathbf{x}, t)=v_{i}\left(\mathbf{x}+L \mathbf{e}_{j}, t\right), \quad t \geq 0 \\
\int_{\Omega} \mathbf{v}(\mathbf{x}, t) d \mathbf{x}=0
\end{gathered}
$$

For the initial-boundary value problem (1.8)-(1.11), Bellout, Bloom and Nečas [17], [18] have established the following result on existence and uniqueness of solutions:

Theorem $1.1([17],[18])$. Let $H(\Omega)=\left\{\mathbf{v} \in L^{2}(\Omega) \mid \nabla \cdot \mathbf{v}=0\right\}$. If $\mathbf{v}_{0} \in H$ then there exists a unique solution $\mathbf{v}$ of the problem (1.8)-(1.11) such that $\forall t_{0}>0, \mathbf{v} \in$ $L^{\infty}([0, \infty) ; H) \cap L^{\infty}\left(\left(t_{0}, \infty\right) ; H^{2}(\Omega)\right)$. Furthermore, the solution operators $S(t): \mathbf{v}_{0} \rightarrow$ $\mathbf{v}(t)$ are $C^{0}$ maps from $H$ into $H, \forall t \geq 0$. In fact, $\mathbf{v} \in C([0, T) ; H), \forall T>0$, so that $S(t)$ yields a nonlinear semigroup.

For the spatially periodic problem, i.e., (1.8), (1.9), (1.10), (1.12a,b), with $\mu_{1}>0$, a result analogous to the above theorem follows quite easily; these results contrast quite 
sharply with what is known about the solutions of the Navier-Stokes equations in space dimension $n=3$, where there exists a unique strong solution only on some interval $\left[0, t_{1}\right]$, $t_{1}=t_{1}\left(\left\|\mathbf{v}_{0}\right\|_{L^{2}}\right)$, while a weak solution exists $\forall t>0$ and agrees with the strong solution on $\left[0, t_{1}\right]$, but may not be unique.

For the space-periodic version of the bipolar viscous problem in dimensions $n=2,3$, with $\mu_{1}=0$, Bellout, Bloom, and Nečas [18] have established the following results, where $p=2-\alpha$ :

TheOrEm 1.2. Consider the problem (1.8), (1.9), (1.10) with $\mu_{1}=0$ in $\Omega=[0, L]^{n}$, $n=2,3$, subject to the spatial periodicity conditions $(1.12 \mathrm{a}, \mathrm{b})$.

(i) For $\frac{3}{2}<p<2 \Leftrightarrow 0<\alpha<\frac{1}{2}$, in $\operatorname{dim} n=2$, or for $\frac{9}{5}<p<\frac{11}{5} \Leftrightarrow-\frac{1}{5}<\alpha<\frac{1}{5}$, in $\operatorname{dim} n=3$, there exists a weak solution (which is probably not unique).

(ii) For $p \geq 2 \Leftrightarrow \alpha \leq 0$, in $\operatorname{dim} n=2$, or for $p \geq \frac{11}{5} \Leftrightarrow \alpha \leq-\frac{1}{5}$, in $\operatorname{dim} n=3$, there exists a unique regular weak solution (i.e., a unique solution in $L^{p}\left([0, T) ; W^{1 . p}(\Omega)\right) \cap$ $L^{\infty}\left([0, T) ; W_{\text {per }}^{1,2}(\Omega)\right)$.

(iii) For $p>1 \Leftrightarrow \alpha<1$, in $\operatorname{dim} n=2$, or for $\frac{6}{5}<p \leq \frac{9}{5} \Leftrightarrow \frac{1}{5} \leq \alpha<\frac{4}{5}$, in $\operatorname{dim} n=3$, there exists a unique Young measure-valued solution.

The results delineated in Theorem 1.2 for the spatially periodic case with $\mu_{1}=0$ have also been summarized in [19] and may be compared with those obtained by Ladyzhenskaya [18], [9], who proved (again for $\mu_{1}=0$ in (1.8), (1.9), (1.10), but in a bounded domain $\Omega$ with $\mathbf{v}=0$ on $\partial \Omega \times[0, T)$ ) the existence of a weak solution for $p>1+\frac{2 n}{n+2}$ and the existence of a unique weak solution for $p \geq 2$ in $\operatorname{dim} n=2$, and for $p \geq \frac{5}{2}$ in $\operatorname{dim} n=3$. Du and Gunzburger [11] claim to have proven the existence of a unique weak solution for the same problem in $\operatorname{dim} n=3$, in the bounded domain scenario, for $p \geq \frac{11}{5}$, but the proof of one of their key results appears to be flawed.

The existence proofs in [17], [18], [19] depend, in part, on two Korn-type inequalities as given by

Theorem 1.3. (i) For $\mathbf{v} \in W_{0}^{1,2}(\Omega) \cap W^{2,2}(\Omega), \Omega \subseteq R^{n}, n=2,3$ with sufficiently smooth boundary $\partial \Omega$,

$$
\int_{\Omega} \frac{\partial e_{i j}}{\partial x_{k}} \frac{\partial e_{i j}}{\partial x_{k}} d \mathbf{x} \geq k_{1}(\Omega)|\mathbf{v}|_{H^{2}(\Omega)}^{2}
$$

for some $k_{1}(\Omega)>0$.

(ii) For $\mathbf{v} \in W^{1, p}(\Omega)$

$$
\left(\int_{\Omega}\left[e_{i j}(\mathbf{v}) e_{i j}(\mathbf{v})\right]^{p / 2} d \mathbf{x}\right)^{2 / p} \geq k_{2}(\Omega)|\mathbf{v}|_{W^{1 \cdot p}(\Omega)}^{2}
$$

for some $k_{2}(\Omega)>0$.

The proof of part (i) of Theorem 1.3 may be found in the appendix to [17], while part (ii) is generally attributed to Nečas [20].

Finally, we note that special examples of flows of incompressible bipolar viscous fluids have been examined in detail in [5] as well as in the recent series of papers by Bellout and Bloom on plane Poiseuille flows ([21]-[23]) and the study by Bloom and Hao [24] of Couette flow between rotating cylinders. 
From the aforementioned existence and uniqueness theorems proven for the incompressible, nonlinear, bipolar initial-boundary value problem, in the case where $\mu_{1}>0$ (and we consider in $\operatorname{dim} n=2,3$ either the associated boundary data (1.11) or the spatial periodicity conditions $(1.12 \mathrm{a}, \mathrm{b}))$, it follows that the solution operator $S(t)$ yields a nonlinear semigroup; the nature of the orbits of this semigroup, as $t \rightarrow \infty$, has been investigated in a series of papers [25]-[27], where it is proven that the problem admits absorbing sets $B_{H}^{\rho}$, a ball of radius $\rho$ in $H(\Omega)$, and $B_{H^{2}}^{\rho^{\prime}}$, a ball of radius $\rho^{\prime}$ in $H_{*}^{2}(\Omega)=\left\{\mathbf{u} \in H^{2}(\Omega) \mid \nabla \cdot \mathbf{u}=0\right\}$. We recall (see, e.g., Temam [15]) that a set $B \subset H(\Omega)$ is absorbing in $J \subseteq H(\Omega)$ if, for every bounded set $B_{0} \subset J, \exists t_{0}=t_{0}\left(B_{0}\right)$ such that $S(t) B_{0} \subset B$ for $t \geq t_{0}\left(B_{0}\right)$ with the analogous definition for absorbing sets in $H_{*}^{2}(\Omega)$. The existence of the absorbing sets $B_{H}^{\rho}$ and $B_{H_{*}^{\prime}}^{\rho^{\prime}}\left(\rho^{\prime}\right.$ depends on $\mu_{1}>0$ and $\rho^{\prime} \rightarrow+\infty$ as $\mu_{1} \rightarrow 0^{+}$) enabled the authors in [25] to prove that the set

$$
\mathcal{A}=\bigcap_{t>0} S(t) B_{H_{*}^{2}(\Omega)}^{\rho^{\prime}}
$$

is a maximal compact global attractor for the orbits of the bipolar equations when $0 \leq$ $\alpha<1$ and $\mu_{1}>0$. By an attractor for the orbits of the bipolar equations we mean, of course, a set $\mathcal{A}$ which satisfies $S(t) \mathcal{A}=\mathcal{A}, \forall t \geq 0$, and $\operatorname{dist}\left(S(t) \mathbf{v}_{0}, \mathcal{A}\right) \rightarrow 0$ as $t \rightarrow \infty$, for all $\mathbf{v}_{0}$ in some neighborhood of $A$. We note that the existence of the absorbing set $B_{H_{*}^{2}}^{\rho^{\prime}}$ enables one to deduce the uniform compactness of the semigroup $S(t)$ for large $t$.

For the space-periodic problem associated with (1.8)-(1.10), it is known (Theorem 1.2) that, if $\mathbf{v}_{0} \in W_{\text {per }}^{1,2}(\Omega)$, there exists a unique solution $\mathbf{v}$ in $C\left([0, T) ; W_{0}^{1,2}(\Omega)\right), \forall T>0$, for $p \geq 2$ in $\operatorname{dim} n=2$, and for $p \geq \frac{11}{5}$ in $\operatorname{dim} n=3$, when $\mu_{1}=0$. In [26], [27], Bloom has established the existence in $\operatorname{dim} n=2$, for the spatially periodic case, of absorbing sets $B_{W_{\text {per }}^{1.2}(\Omega)}^{\bar{\rho}}$ in $W_{\text {per }}^{1,2}(\Omega)$ for the bipolar problem $\left(\mu_{1}>0\right)$ which are, in fact, independent of $\mu_{1}$ when $p \geq 2$; these sets are then also absorbing sets in $W_{\text {per }}^{1,2}(\Omega)$, when $\operatorname{dim} n=2$, for the non-Newtonian problem $\left(\mu_{1}=0\right)$ with $p \geq 2$. With $\mu_{1}>0$ and $p>2$, we also have the existence of absorbing sets in $H_{\text {per }}(\Omega)$ and $V_{\text {per }}(\Omega)$, where

$$
\begin{aligned}
& H_{\text {per }}(\Omega)=\left\{\mathbf{u}: \Omega \rightarrow R^{n} \mid \mathbf{u} \in L_{\text {per }}^{2}(\Omega) ; \nabla \cdot \mathbf{u}=0, \int_{\Omega} \mathbf{u} d \mathbf{x}=\mathbf{0}\right\} \\
& V_{\text {per }}(\Omega)=\left\{\mathbf{u}: \Omega \rightarrow R^{n} \mid \mathbf{u} \in H_{\text {per }}^{2}(\Omega) ; \nabla \cdot \mathbf{u}=0, \int_{\Omega} \mathbf{u} d \mathbf{x}=\mathbf{0}\right\}
\end{aligned}
$$

and, as a consequence, the existence of attractors $\mathcal{A}_{\mu_{1}}, \mu_{1}>0$, and $\mathcal{A}_{0}$ which are given by

$$
\left\{\begin{array}{l}
\mu_{1}>0: \mathcal{A}_{\mu_{1}}=\bigcap_{t>0} S_{\mu_{1}}(t) B_{H_{\text {per }}^{2}(\Omega)}^{\rho^{\prime}\left(\mu_{1}\right)}, \\
\mu_{1}>0: \mathcal{A}_{0}=\bigcap_{t>0} S_{0}(t) B_{W_{\text {per }}^{1,2}(\Omega)}^{\bar{\rho}} .
\end{array}\right.
$$

In (1.17) the dependence on $\mu_{1}$ is now explicitly displayed and both the cases $\mu_{1}=0$ and $\mu_{1}>0$ refer to the situation where $\operatorname{dim} n=2, p \geq 2$, and the spatial periodicity conditions apply; it is then possible to draw some conclusions about the relation between 
$\mathcal{A}_{\mu_{1}}$ and $\mathcal{A}_{0}$. In fact, it is proven in [26], [27] that if $d$ denotes the semidistance for sets, then, as $\mu_{1} \rightarrow 0^{+}$

$$
d\left(\mathcal{A}_{0}, \mathcal{A}_{\mu_{1}}\right)=\sup _{\mathbf{x} \in \mathcal{A}_{0}} \inf _{\mu_{1} \in \mathcal{A}_{\mu_{1}}}\left|\mathbf{x}-\mathbf{y}_{\mu_{1}}\right|_{L^{2}(\Omega)} \rightarrow 0
$$

thus establishing the upper semicontinuity of the attractor $\mathcal{A}_{\mu_{1}}$, with respect to the higher-order viscosity parameter $\mu_{1}$, when $p \geq 2$, in the spatially periodic case.

For a fixed, arbitrary $\mu_{1}>0$, with $0 \leq \alpha<1(\Leftrightarrow 1<p \leq 2)$ it has been demonstrated in [25] that

(i) the $S_{\mu_{1}}(t)$ are uniformly differentiable on the attractor $\mathcal{A}_{\mu_{1}}, \forall t \geq 0$, and

(ii) the linear operators $\mathbf{L}\left(t ; \mathbf{u}_{0}\right): \mathbf{J} \rightarrow \mathbf{U}(t), \mathbf{U}(t)$ the unique solution of the linearized bipolar problem in $L^{\infty}([0, \infty) ; H(\Omega))$ satisfying $\mathbf{U}(0)=\mathbf{J} \in H(\Omega)$, with the linearization taken about $\mathbf{u}(t)=S(t) \mathbf{u}_{0}$, are uniformly bounded, $\forall t \geq 0$, in $\mathcal{L}(H(\Omega) ; H(\Omega))$, when $\mathbf{u}_{0} \in \mathcal{A}_{\mu_{1}}$.

The uniform differentiability of the $S_{\mu_{1}}(t)$ in [25] is established by showing that

$$
\sup _{\substack{\mathbf{u}_{0}, \mathbf{v}_{0} \in \mathcal{A}_{\mu_{1}} \\ 0<\left|\mathbf{v}_{0}-\mathbf{u}_{o}\right|<\epsilon}}\left(|\Theta(t)|_{L^{2}} /\left|\mathbf{v}_{0}-\mathbf{u}_{0}\right|_{L^{2}}\right) \rightarrow 0 \quad \text { as } \epsilon \rightarrow 0,
$$

where $\boldsymbol{\Theta}(t)=S_{\mu_{1}}(t) \mathbf{v}_{0}-S_{\mu_{1}}(t) \mathbf{u}_{0}-\mathcal{L}\left(t ; \mathbf{u}_{0}\right)\left(\mathbf{v}_{0}-\mathbf{u}_{0}\right)$. The uniform boundedness of the linear operators $\mathcal{L}\left(t ; \mathbf{u}_{0}\right)$ is established by examining the behavior of the solution of the linearized problem, i.e., of

$$
\begin{aligned}
\frac{\partial U_{i}}{\partial t} & +U_{j} \frac{\partial u_{i}}{\partial x_{j}}+v_{j} \frac{\partial U_{i}}{\partial x_{j}} \\
& =-\frac{\partial p}{\partial x_{i}}-2 \mu_{1} \frac{\partial}{\partial x_{j}}\left(\Delta e_{i j}(\mathbf{U})\right)+\frac{\partial}{\partial x_{j}}\left[\frac{\partial^{2} \Gamma}{\partial e_{i j} \partial e_{k l}}(\mathbf{e}(\mathbf{u})) e_{k l}(\mathbf{U})\right]
\end{aligned}
$$

$\mathbf{u}(t)=S_{\mu_{1}}(t) \mathbf{u}_{0}, \mathbf{u}_{0} \in \mathcal{A}_{\mu_{1}}$, and

$$
\Gamma\left(e_{y} e_{i j}\right)=\int_{0}^{e_{i j} e_{i, j}} \mu_{0}(\epsilon+s)^{-\alpha / 2} d s
$$

subject to

$$
\begin{cases}\nabla \cdot \mathbf{U}=0, & \text { in } \Omega \times[0, T), \\ \mathbf{U}(\mathbf{x}, 0)=\mathbf{U}_{0}(\mathbf{x}), & \text { in } \Omega, \\ U_{i}=\boldsymbol{\tau}_{i j k}(\mathbf{U}) \nu_{j} \nu_{k}=0, & \text { on } \partial \Omega \times[0, T) .\end{cases}
$$

Once the uniform differentiability of $S_{\mu_{1}}(t)$ on the attractor $\mathcal{A}_{\mu_{1}}$, and the uniform boundedness of the linearized operators $\mathcal{L}\left(t ; \mathbf{u}_{0}\right), \mathbf{u}_{0} \in \mathcal{A}_{\mu_{1}}$, have been established, it is then possible to use the framework developed by Constantin, Foiaş, and Temam (see, e.g., [15], [28], or [29]) in order to deduce upper bounds for both the Hausdorff and fractal dimensions, $d_{H}\left(\mathcal{A}_{\mu_{1}}\right)$ and $d_{F}\left(\mathcal{A}_{\mu_{1}}\right)$, respectively, of the global compact attractor $\mathcal{A}_{\mu_{1}}$; it is, in fact, shown in [25] that for $0 \leq \alpha<1(\Leftrightarrow 1<p \leq 2)$ and $\mu_{1}>0$, in space $\operatorname{dim} n=3, d_{H}\left(\mathcal{A}_{\mu_{1}}\right) \leq k$ and $d_{F}\left(\mathcal{A}_{\mu_{1}}\right) \leq 2 k$, with $k$ the first positive integer such that

$$
k-1<\frac{m(\Omega)|\mathbf{f}|_{\infty}^{3}}{\left(\lambda_{1} \Lambda\right)^{3 / 2} \mu_{1}^{9 / 12}}<k .
$$


In $(1.23), m(\Omega)$ is a generic constant arising through the implementation of various embeddings and interpolation-type estimates, $|\mathbf{f}|_{\infty}=|\mathbf{f}|_{L^{\infty}\left([0, \infty) ; L^{2}(\Omega)\right)}, \lambda_{1}$ is the smallest eigenvalue of $-\Delta$ on $\Omega$, and $\Lambda \rightarrow 0$ as $\mu_{1} \rightarrow 0$.

The same type of analysis as that referenced above has been used for the bipolar problem $\left(\mu_{1}>0\right)$ in both $\operatorname{dim} n=2,3$, when $p \geq 2$, to establish upper bounds for $d_{H}\left(\mathcal{A}_{\mu_{1}}\right)$ and $d_{F}\left(\mathcal{A}_{\mu_{1}}\right)$ which are in fact independent of $\mu_{1}$; such a procedure for the case $\mu_{1}=0, p \geq 2$, even in $\operatorname{dim} n=2$ with the spatial periodicity conditions, does not appear possible, as it does not seem to be true that $S_{0}(t)$ is uniformly differentiable on the attractor $\mathcal{A}_{0}$. Furthermore, even though the bounds obtained for $d_{H}\left(\mathcal{A}_{\mu_{1}}\right)$ and $d_{F}\left(\mathcal{A}_{\mu_{1}}\right)$ are independent of $\mu_{1}>0$, the upper semicontinuity result represented by (1.18) is not strong enough to enable one to deduce an upper bound for, say, $d_{H}\left(\mathcal{A}_{0}\right)$ by looking at the limit $\lim _{\mu_{1} \rightarrow 0^{+}}$of the relevant upper bound for $d_{H}\left(\mathcal{A}_{\mu_{1}}\right)$; this problem of determining upper bounds for the Hausdorff and fractal dimensions of the global attractor $\mathcal{A}_{0}$ for the non-Newtonian $\left(\mu_{1}=0\right)$ problem, when $p \geq 2$ (even in space $\operatorname{dim} n=2$ with the assumption of spatial periodicity), appears to still be open and is the subject of current work by Malek and Nečas [30]. Another open problem in the dynamical systems approach to the large time behavior of solutions of the incompressible bipolar equations is that of the existence of an inertial manifold; it is on that problem that our attention will be focused in this paper.

The concept of an inertial manifold for dissipative nonlinear evolutionary equations appears to have been introduced in Foiaş, Sell, and Temam [31]. Formally, an inertial manifold is a finite-dimensional attractor which attracts exponentially all orbits of the evolutionary equation in question. Denoting again by $S_{\mu_{1}}(\cdot)$ the solution operator for the bipolar problem $\left(\mu_{1}>0\right.$ and $\left.0 \leq \alpha<1 \Leftrightarrow 1<p \leq 2\right)$ (1.8), (1.9), (1.10) satisfying the spatial periodicity conditions $(1.12 \mathrm{a}, \mathrm{b})$ on $\Omega=[0, L]^{n}, L>0, n=2,3$, we have the following:

Definition 1.1. A set $\mathcal{M}$ is an inertial manifold for the bipolar problem on $\Omega \times[0, T)$, $T>0$, with solution operator $S_{\mu_{1}}(\cdot)$, if

(i) $\mathcal{M}$ is a finite-dimensional Lipschitz manifold,

(ii) $\mathcal{M}$ is invariant in the sense that $\forall t \geq 0, S_{\mu_{1}}(t) \mathcal{M} \subset \mathcal{M}$, and

(iii) $\mathcal{M}$ attracts exponentially all orbits of $S_{\mu_{1}}(\cdot)$, i.e.,

$$
\operatorname{dist}\left(S_{\mu_{1}}(t) \mathbf{v}_{0}, \mathcal{M}\right) \rightarrow 0 \quad \text { as } t \rightarrow \infty
$$

The convergence indicated in (1.24) holds $\forall \mathbf{v}_{0} \in \mathcal{D}\left(\mathbf{A}^{1 / 4}\right)$, where $\mathbf{A}$ is the linear selfadjoint positive operator associated with the highest-order derivative terms in (1.8); in fact, (1.24) holds $\forall \mathbf{v}_{0} \in H_{\text {per }}(\Omega)$ and the rate of decay is uniformly exponential in (1.24) for $\mathbf{v}_{0}$ in bounded sets of $\mathcal{D}\left(\mathbf{A}^{1 / 4}\right)$ with $\mathcal{M} \subseteq \mathcal{D}\left(\mathbf{A}^{1 / 4}\right)$. In the next section we will specify the structure of the operator $\mathbf{A}$ and recast the problem (1.8)-(1.10), (1.12 a,b) as an initial-value problem for a nonlinear evolution equation in the Hilbert space $V_{\text {per }}(\Omega), \Omega=[0, L]^{n}, n=2,3$. In $\S 3$ we provide a broad and specific outline of all the key results to be proved in subsequent sections, culminating in the theorem which implies the existence of an inertial manifold for the bipolar problem.

The subject of inertial manifolds for dissipative evolutionary equations has attracted considerable interest since the initial work by Foiaş, Sell, and Temam [31], and there is 
now an extensive literature. We may refer the interested reader to the monographs [32], [33] as well as to the recent papers [34]-[49]; many of these papers deal with efforts to construct inertial manifolds for the Navier-Stokes equations or some ad hoc regularization thereof (i.e., an approximate inertial manifold). A critical discourse on the use of approximate inertial manifolds for the Navier-Stokes model in the modeling of turbulence phenomena may be found in the paper of Heywood and Rannacher [49]. For related work on the existence of attractors for nonlinear evolution equations, and estimates of their dimension, with a particular emphasis on problems arising in viscous fluid flow (including turbulence), we may direct the reader to the excellent surveys by Temam [15], Hale [50], and Babin and Vishik [52], as well as to the recent papers [56]-[62].

2. Formulation of the problem in Hilbert space. We begin by explicitly writing out the system of equations in the form

$$
\begin{aligned}
\frac{\partial \mathbf{v}}{\partial t}+\mathbf{v} \cdot \nabla \mathbf{v}= & -\nabla \bar{p}-2 \mu_{1} \nabla \cdot(\Delta \mathbf{e}) \\
& +\nabla \cdot\left(2 \mu_{0}\left(\epsilon+|\mathbf{e}(\mathbf{v})|^{2}\right)^{(p-2) / 2} \mathbf{e}\right)+\mathbf{f}
\end{aligned}
$$

In (2.1) we have set $\alpha=2-p$, with $1 \leq p \leq 2$ for $0 \leq \alpha<1$, have denoted the pressure field by $\bar{p}$, to distinguish it from the index $p$, and have, without loss of generality, set the constant density $\rho=1$. We are considering the system (2.1), coupled with the incompressibility constraint (1.9) and the initial data (1.10), for the space-periodic situation in which $(1.12 \mathrm{a}, \mathrm{b})$ hold for some $L>0$, so that our basic function spaces are $H_{\text {per }}(\Omega)$ and $V_{\text {per }}(\Omega), \Omega=[0, L]^{n}, n=2,3$, as given by (1.16). We introduce the following fundamental linear problem associated with $(2.1),(1.9),(1.10),(1.12 \mathrm{a}, \mathrm{b})$ : find $(\mathbf{u}, \bar{p}): \Omega \rightarrow R^{n} \times R$ such that

$$
\begin{gathered}
\nabla \cdot(\Delta \mathbf{e})+\nabla \bar{p}=f, \quad \text { in } \Omega, \\
\nabla \cdot \mathbf{u}=0, \quad \text { in } \Omega,
\end{gathered}
$$

where $\mathbf{e}=\mathbf{e}(\mathbf{u})$ and $\mathbf{u}$ satisfies the periodicity conditions (1.12a,b). Associated with the problem $(2.2 \mathrm{a}, \mathrm{b}),(1.12 \mathrm{a}, \mathrm{b})$ is the linear operator $\mathbf{A}$ which is defined as follows: consider the positive definite $V_{\text {per }}(\Omega)$-elliptic symmetric bilinear form $a(\cdot, \cdot): V_{\text {per }} \times V_{\text {per }} \rightarrow R$ given by

$$
a(\mathbf{u}, \mathbf{v})=\frac{1}{2}(\Delta \mathbf{u}, \Delta \mathbf{v})
$$

As a consequence of the Lax-Milgram lemma [12] we obtain an isometry $A \in \mathcal{L}\left(V_{\text {per }} ; V_{\text {per }}^{\prime}\right)$, $V_{\text {per }}^{\prime}$ the dual of $V_{\text {per }}$, via

$$
\langle\mathbf{A u}, \mathbf{v}\rangle_{V_{\text {per }}^{\prime} \times V_{\text {per }}}=a(\mathbf{u}, \mathbf{v})=\langle\mathbf{f}, \mathbf{v}\rangle_{V_{\text {per }}^{\prime} \times V_{\text {per }}^{\prime}}, \quad \forall \mathbf{v} \in V_{\text {per }}
$$

with $\mathbf{f} \in V_{\text {per. }}^{\prime}$. For the domain of $\mathbf{A}$ we have

$$
D(\mathbf{A})=\left\{\mathbf{u} \in V_{\text {per }} \mid a(\mathbf{u}, \mathbf{v})=(\mathbf{f}, \mathbf{v})_{L^{2}(\Omega)}, \mathbf{f} \in H_{\text {per }} \subset V_{\text {per }}^{\prime}, \forall \mathbf{v} \in V_{\text {per }}\right\} .
$$

In all that follows, $(\cdot, \cdot)$ without a subscript will denote $(\cdot, \cdot)_{L^{2}(\Omega)}$. Thus $\mathbf{A} \in \mathcal{L}(D(\mathbf{A})$; $\left.H_{\text {per }}\right) \cap \mathcal{L}\left(V_{\text {per }}, V_{\text {per }}^{\prime}\right)$. As a consequence of Rellich's lemma [12], $\mathbf{A}^{-1}$ is compact as a 
mapping in $V_{\text {per }}^{\prime}$ (or in $H_{\text {per }}$ ). Therefore, the spectrum of $\mathbf{A}$ consists of real eigenvalues $\lambda_{j}$, with the multiplicity of each $\lambda_{j}$ finite; these eigenvalues $\lambda_{j}$ may be ordered, i.e.,

$$
0<\lambda_{1} \leq \lambda_{2} \leq \cdots, \quad \lambda_{j} \rightarrow+\infty \quad \text { as } j \rightarrow+\infty
$$

and the only possible accumulation point of $\left\{\lambda_{j}\right\}$ is at infinity. The selfadjoint operator A possesses an orthonormal set of eigenfunctions $\left\{\phi_{j}\right\}_{j=1}^{\infty}$, which is complete in $V_{\text {per }}^{\prime}$ (or $\left.H_{\text {per }}\right)$, and which satisfies

$$
\mathbf{A} \phi_{j}=\lambda_{j} \phi_{j}, \quad \text { with } \phi_{j} \in V_{\text {per }}(\text { or } \mathbf{D}(\mathbf{A})), \forall j
$$

Because $\mathbf{A}^{-1}$ is compact, we can define the fractional powers of $\mathbf{A}$ by using the spectral resolution of $\mathbf{A}$ as follows: $\forall \alpha \in R$ we define

$$
\mathbf{A}^{\alpha} \mathbf{u}=\sum_{k=1}^{\infty} \lambda_{k}^{\alpha}\left(\mathbf{u}, \phi_{k}\right) \phi_{k}, \quad \forall \mathbf{u} \in D\left(\mathbf{A}^{\alpha}\right),
$$

where for $\alpha>0$

$$
D\left(\mathbf{A}^{\alpha}\right)=\left\{\mathbf{u} \in H_{\text {per }} \mid \sum_{j=1}^{\infty} \lambda_{j}^{2 \alpha}\left(\mathbf{u}, \phi_{j}\right)^{2}<\infty\right\},
$$

while for $\alpha<0, D\left(\mathbf{A}^{\alpha}\right)$ is the completion of $H_{\text {per }}$ with respect to the norm

$$
\|\mathbf{u}\|_{\alpha}=\left\{\sum_{j=1}^{\infty} \lambda_{j}^{2 \alpha}\left(\mathbf{u}, \phi_{j}\right)^{2}\right\}^{1 / 2}
$$

which is induced by the scalar product

$$
(\mathbf{u}, \mathbf{v})_{D\left(\mathbf{A}^{\alpha}\right)}=\sum_{j=1}^{\infty} \lambda_{j}^{2 \alpha}\left(\mathbf{u}, \boldsymbol{\phi}_{j}\right)\left(\mathbf{v}, \boldsymbol{\phi}_{j}\right)
$$

Using the Fourier transform, it is a straightforward exercise to show that there exist constants $k_{1}, k_{2}>0$ such that

$$
\begin{array}{cl}
k_{1}\|\mathbf{u}\|_{H^{4}(\Omega)} \leq|\mathbf{A} \mathbf{u}|_{L^{2}(\Omega)} \leq k_{2}\|\mathbf{u}\|_{H^{4}(\Omega)}, & \forall \mathbf{u} \in D(\mathbf{A}), \\
k_{1}\|\mathbf{u}\|_{H^{2}(\Omega)} \leq\left|\mathbf{A}^{1 / 2} \mathbf{u}\right|_{L^{2}(\Omega)} \leq k_{2}\|\mathbf{u}\|_{H^{2}(\Omega)}, & \forall \mathbf{u} \in D\left(\mathbf{A}^{1 / 2}\right),
\end{array}
$$

and

$$
k_{1}\|\mathbf{u}\|_{H^{1}(\Omega)} \leq\left|\mathbf{A}^{1 / 4} \mathbf{u}\right|_{L^{2}(\Omega)} \leq k_{2}\|\mathbf{u}\|_{H^{1}(\Omega)}, \quad \forall \mathbf{u} \in D\left(\mathbf{A}^{1 / 4}\right)
$$

Therefore, we have the equivalences

$$
\left\{\begin{array}{l}
|\mathbf{A u}|_{L^{2}(\Omega)} \sim\|\mathbf{u}\|_{H^{4}(\Omega)}, \\
\left|\mathbf{A}^{1 / 2} \mathbf{u}\right|_{L^{2}(\Omega)} \sim\|\mathbf{u}\|_{H^{2}(\Omega)} \\
\left|\mathbf{A}^{1 / 4} \mathbf{u}\right|_{L^{2}(\Omega)} \sim\|\mathbf{u}\|_{H^{1}(\Omega)}
\end{array}\right.
$$


For the space-periodic problem it is easy to show that the eigenvalues of $\mathbf{A}$ have the form

$$
\lambda_{\mathbf{k}}=\frac{8 \pi^{4}}{L^{4}}|\mathbf{k}|^{4}, \quad \mathbf{k}=\left(k_{1}, \ldots, k_{n}\right)
$$

for $n=2,3$. An extended discussion of the eigenvalue problem (2.7) may be found in the Appendix.

We now set, for $\mathbf{e}=\mathbf{e}(\mathbf{u})$,

$$
\begin{gathered}
{\left[\mathbf{A}_{p} \mathbf{u}\right]_{i}=\frac{\partial}{\partial x_{j}}\left[\left(\epsilon+|\mathbf{e}|^{2}\right)^{(p-2) / 2} e_{i j}\right], \quad i=1, \ldots, n} \\
\mathbf{B}(\mathbf{u}, \mathbf{v})=\mathbf{u} \cdot \nabla \mathbf{v}
\end{gathered}
$$

and

$$
\mathbf{R}(\mathbf{u})=-2 \mu_{0} \mathbf{A}_{p}(\mathbf{u})+\mathbf{B}(\mathbf{u}, \mathbf{u})-\mathbf{f},
$$

where we take $\mathbf{f} \in H_{\text {per. }}$. Then the bipolar problem (2.1), (1.9), (1.10), (1.12a,b) can be viewed as an initial-value problem for an equation of evolution in the Hilbert space $V_{\text {per }}$, namely,

$$
\begin{gathered}
\frac{d \mathbf{u}}{d t}+2 \mu_{1} \mathbf{A} \mathbf{u}+\mathbf{R}(\mathbf{u})=\mathbf{0}, \quad t>0 \\
\mathbf{u}(\mathbf{0})=\mathbf{u}_{0} \in H_{\text {per }} .
\end{gathered}
$$

In $\S 3$ we will map out the steps that are involved in proving the existence of an inertial manifold for (2.18), (2.19).

3. An outline of the basic methodology. We delineate, in this section, the sequence of results that will lead to the proof of the existence of an inertial manifold for the initial-value problem associated with the evolution equation (2.18) in the Hilbert space $V_{\text {per }}$. The basic ideas are as follows:

I. We first prove a Lipschitz property for the nonlinear map $\mathbf{R}(\cdot)$; in fact, we will show that $\mathbf{R}(\mathbf{u})$ is Lipschitz on bounded sets of $D\left(\mathbf{A}^{1 / 4}\right)$ with values in $D\left(\mathbf{A}^{-1 / 4}\right)$, i.e., for $M>0, \exists C_{M}>0$ such that

$$
\left|\mathbf{A}^{-1 / 4} \mathbf{R}(\mathbf{u})-\mathbf{A}^{-1 / 4} \mathbf{R}(\mathbf{v})\right| \leq C_{M}\left|\mathbf{A}^{1 / 4}(\mathbf{u}-\mathbf{v})\right|
$$

$\forall \mathbf{u}, \mathbf{v} \in D\left(\mathbf{A}^{1 / 4}\right)$ such that $\left|\mathbf{A}^{1 / 4}(\mathbf{u})\right| \leq M$ and $\left|\mathbf{A}^{1 / 4}(\mathbf{v})\right| \leq M$, where, as in the remainder of this paper, $|\cdot|$ means the $L^{2}$ norm $|\cdot|_{L^{2}(\Omega)}, n=2,3, \Omega=[0, L]^{n}, L>0$. The result expressed by (3.1) will be stated as a formal theorem and proven in $\S 4$; it clearly implies that $\mathbf{R}$ is bounded on bounded subsets of $D\left(\mathbf{A}^{1 / 4}\right)$, i.e., that $\exists C_{M}^{\prime}>0$ such that

$$
\left|\mathbf{A}^{-1 / 4} \mathbf{R}(\mathbf{u})\right| \leq C_{M l}^{\prime}, \quad \forall \mathbf{u} \in D\left(\mathbf{A}^{1 / 4}\right) \text { with }\left|\mathbf{A}^{1 / 4} \mathbf{u}\right| \leq M
$$

II. Using the Lipschitz property (3.1) we will establish, in $\S 5$, a squeezing property for orbits of the semigroup generated by $(2.18),(2.19)$ of the following form. Let $\mathbf{w}_{1}, \ldots, \mathbf{w}_{N}$ be the first $N$ eigenfunctions of the operator $\mathbf{A}$. Let $\mathbf{P}_{N}: H_{\text {per }} \rightarrow \operatorname{span}\left\{\mathbf{w}_{1}, \ldots, \mathbf{w}_{N}\right\}$ 
be the usual projection operator and set $\mathbf{Q}_{N}=\mathbf{I}-\mathbf{P}_{N}$. Let $\gamma>0$. Then we will prove that if, for $t \in[0, T], T>0$,

$$
\left|\mathbf{A}^{1 / 4}(\mathbf{u}(t))\right| \leq M \quad \text { and } \quad\left|\mathbf{A}^{1 / 4}(\mathbf{v}(t))\right| \leq M
$$

$M>0$, as in the statement of the Lipschitz property (3.1), then $\exists c_{i}>0, i=1,2$, depending only on $\gamma, M, T, \mathbf{f}, \mu_{0}, \mu_{1}, \epsilon$, and $\Omega$, such that for every $N$ and every $t \in[0, T]$ either

$$
\left|\mathbf{Q}_{N} A^{-1 / 4}(\mathbf{u}(t)-\mathbf{v}(t))\right| \leq \gamma\left|\mathbf{P}_{N} \mathbf{A}^{-1 / 4}(\mathbf{u}(\mathbf{t})-\mathbf{v}(t))\right|
$$

or else

$$
\left|\mathbf{A}^{-1 / 4}(\mathbf{u}(t)-\mathbf{v}(t))\right| \leq c_{1} \exp \left(-c_{2} \mu_{1} \gamma_{N+1} t\right)\left|\mathbf{A}^{-1 / 4}(\mathbf{u}(0)-\mathbf{v}(0))\right| .
$$

We remark in passing that an $L^{2}$ version of the squeezing property expressed by (3.4), (3.5) also holds relative to the orbits of the semigroup generated by $(2.18),(2.19)$ but is not well-adapted to the proof of the existence of an inertial manifold; the $L^{2}$ version of the squeezing property for the (space-periodic) bipolar problem has been established by the authors in [68].

III. From the analysis in [25] it follows that there exist absorbing balls in $H_{\text {per }}$, $D\left(\mathbf{A}^{1 / 4}\right)$, and $V_{\text {per }}$ which attract all the orbits of $(2.18),(2.19)$. We will want, however, in the subsequent discussion to restrict our attention to the dynamics inside an absorbing ball $B_{r_{1}} \subseteq D\left(\mathbf{A}^{1 / 4}\right)$; to this end we shall introduce the smooth cut-off function $\Theta: R^{+} \rightarrow[0,1]$ given by

$$
\begin{cases}\Theta(\xi)=1, & 0 \leq \xi \leq 1, \\ \Theta(\xi)=0, & \xi \geq 2 \\ \left|\Theta^{\prime}(\xi)\right| \leq 2, & \xi \geq 0\end{cases}
$$

and then set $\Theta_{r_{1}}(r)=\Theta\left(r / r_{1}\right)$. We then modify (2.18) and consider, in its place, the evolution equation

$$
\frac{d \mathbf{u}}{d t}+2 \mu_{1} \mathbf{A} \mathbf{u}+\Theta_{r_{1}}\left(\left|\mathbf{A}^{1 / 4} \mathbf{u}\right|\right) \mathbf{R}(\mathbf{u})=\mathbf{0}
$$

It is not difficult to prove the existence and uniqueness of solutions to (3.7), (2.19) with $\mathbf{u}_{0} \in H_{\text {per }}$; in addition, we will show, in $\S 6$, that the ball $B_{r_{2}}, r_{2}=2 r_{1}$, is an absorbing set (for the orbits of $(3.7),(2.19))$ in $D\left(\mathbf{A}^{1 / 4}\right)$; it is also proven, in $\S 6$, that after a sufficiently large time $t^{*}$, the dynamics of the original equation (2.18) are exactly represented by the dynamics of the modified equation (3.7).

IV. In $\S 6$ we introduce the space $H_{b, l}$ of the Lipschitz maps $(b>0, l>0)$

$$
\phi: \mathbf{P}_{N} D\left(\mathbf{A}^{1 / 4}\right) \rightarrow \mathbf{Q}_{N} D\left(\mathbf{A}^{1 / 4}\right)
$$

satisfying

$$
\begin{gathered}
\operatorname{supp} \phi \subset\left\{\mathbf{p} \in \mathbf{P}_{N} D\left(\mathbf{A}^{1 / 4}\right)|| \mathbf{A}^{1 / 4} \mathbf{p} \mid \leq r_{2}\right\} \\
\left|\mathbf{A}^{1 / 4} \boldsymbol{\phi}(\mathbf{p})\right| \leq b, \quad \forall \mathbf{p} \in \mathbf{P}_{N} D\left(\mathbf{A}^{1 / 4}\right)
\end{gathered}
$$


and $\forall \mathbf{p}_{1}, \mathbf{p}_{2} \in \mathbf{P}_{N} D\left(\mathbf{A}^{1 / 4}\right)$

$$
\left|\mathbf{A}^{1 / 4} \phi\left(\mathbf{p}_{1}\right)-\mathbf{A}^{1 / 4} \phi\left(\mathbf{p}_{2}\right)\right| \leq l\left|\mathbf{A}^{1 / 4}\left(\mathbf{p}_{1}-\mathbf{p}_{2}\right)\right| .
$$

It is not difficult to prove that $H_{b, l}$ is complete with respect to the metric induced by the norm

$$
\left\|\phi_{1}-\phi_{2}\right\|=\sup _{\mathbf{p} \in \mathbf{P}_{N} D\left(\mathbf{A}^{1 / 4}\right)}\left|\mathbf{A}^{1 / 4} \boldsymbol{\phi}_{1}(\mathbf{p})-\mathbf{A}^{1 / 4} \boldsymbol{\phi}_{2}(\mathbf{p})\right|
$$

$\phi_{i} \in H_{b, l}, i=1,2$.

V. We next specify a mapping $\mathbf{T}$ which associates with each $\phi \in H_{b . l}$ a function $\mathbf{T} \boldsymbol{\phi}$ defined on $\mathbf{P}_{N} D\left(\mathbf{A}^{1 / 4}\right)$; the mapping $\mathbf{T}$ arises in the following manner:

(i) We apply the projections $\mathbf{P}_{N}, \mathbf{Q}_{N}$ to the modified equation (3.7) to obtain evolution equations for $\mathbf{p}=\mathbf{P}_{N} \mathbf{u}$ and $\mathbf{q}=\mathbf{Q}_{N} \mathbf{u}$ of the form

$$
\left\{\begin{array}{l}
\frac{d \mathbf{p}}{d t}+2 \mu_{1} \mathbf{A} \mathbf{p}+\mathbf{P}_{N} \mathbf{F}(\mathbf{u})=\mathbf{0} \\
\frac{d \mathbf{q}}{d t}+2 \mu_{1} \mathbf{A q}+\mathbf{Q}_{N} \mathbf{F}(\mathbf{u})=\mathbf{0}
\end{array}\right.
$$

where $\mathbf{F}(\mathbf{u})=\Theta_{r_{1}}\left(\left|\mathbf{A}^{1 / 4} \mathbf{u}\right|\right) \mathbf{R}(\mathbf{u})$.

(ii) Next, we choose $\phi \in H_{b . l}$ and $\mathbf{p}_{0} \in \mathbf{P}_{N} D\left(\mathbf{A}^{1 / 4}\right)$ and consider $\mathbf{p}=\mathbf{p}(t)$ as determined by the initial value problem

$$
\left\{\begin{array}{c}
\frac{d \mathbf{p}}{d t}+2 \mu_{1} \mathbf{A p}+\mathbf{P}_{N} \mathbf{F}(\mathbf{p}+\phi(\mathbf{p}))=\mathbf{0} \\
\mathbf{p}(0)=\mathbf{p}_{0}
\end{array}\right.
$$

i.e., $\mathbf{p}(t)=\mathbf{p}\left(t ; \phi, \mathbf{p}_{0}\right)$.

(iii) We employ for our operator $\mathbf{A}$ the following lemma, a proof of which may be found in Temam [15]: for any $\alpha \in R^{1}$, if $\boldsymbol{\sigma} \in L^{\infty}\left(R^{1} ; D\left(\mathbf{A}^{\alpha-1 / 2}\right)\right)$ then $\exists$ ! function $\boldsymbol{\xi}$, continuous and bounded from $R^{1}$ into $D\left(\mathbf{A}^{\alpha}\right)$, which satisfies

$$
\frac{d \boldsymbol{\xi}}{d t}+\mathbf{A} \boldsymbol{\xi}=\boldsymbol{\sigma}
$$

REMARK. In establishing the lemma cited above one looks at the initial value problem

$$
\left\{\begin{array}{l}
\frac{d \boldsymbol{\xi}}{d t}+\mathbf{A} \boldsymbol{\xi}=\mathbf{0} \\
\boldsymbol{\xi}(0)=\boldsymbol{\xi}_{0}
\end{array}\right.
$$

whose (unique) solution $e^{-t \mathbf{A}}: \boldsymbol{\xi}_{0} \rightarrow \boldsymbol{\xi}(t)$ is continuous as a mapping from $D\left(\mathbf{A}^{\alpha-1 / 2}\right)$ into $D\left(\mathbf{a}^{\alpha}\right), \forall t>0$; the unique solution of (3.14) then has the form

$$
\boldsymbol{\xi}(t)=e^{-\left(t-t_{0}\right) \mathbf{A}} \boldsymbol{\xi}\left(t_{0}\right)+\int_{t_{0}}^{t} e^{-(t-\tau) \mathbf{A}} \boldsymbol{\sigma}(\tau) d \boldsymbol{\tau}
$$

and as $\left|e^{-t \mathbf{A}}\right|_{\mathcal{L}\left(D\left(\mathbf{A}^{\alpha}\right)\right)} \leq \exp (-\lambda t)$, for some $\lambda>0$, all $\alpha \in R^{1}$, and all $t \geq 0$, as $t_{0} \rightarrow-\infty$ we obtain

$$
\boldsymbol{\xi}(t)=\int_{-\infty}^{t} e^{-(t-\tau) \mathbf{A}} \boldsymbol{\sigma}(\tau) d \tau
$$


(iv) Having introduced the initial-value problem (3.13) we consider, with $\mathbf{p}=\mathbf{p}\left(t ; \boldsymbol{\phi}, \mathbf{p}_{0}\right)$ as defined by (3.13), the analog of the equation for $\mathbf{p}(t)$, namely,

$$
\frac{d \mathbf{q}}{d t}+2 \mu_{1} \mathbf{A q}+\mathbf{Q}_{N} \mathbf{F}(\mathbf{p}+\boldsymbol{\phi}(\mathbf{p}))=\mathbf{0} .
$$

In (3.18), $\boldsymbol{\sigma} \equiv-\mathbf{Q}_{N} \mathbf{F}(\mathbf{p}+\boldsymbol{\phi}(\mathbf{p})) \in L^{\infty}\left(R^{1} ; D\left(\mathbf{A}^{-1 / 4}\right)\right)$, i.e., (3.18) is of the form (3.14); thus by the lemma referenced in (iii), $\exists$ ! solution $\mathbf{q}=\mathbf{q}\left(t ; \boldsymbol{\phi}, \mathbf{p}_{0}\right)$ of $(3.18)$ which is continuous and bounded as a mapping from $R^{1}$ into $\mathbf{Q}_{N} D\left(\mathbf{A}^{1 / 4}\right)$. In particular,

$$
\mathbf{q}(0)=\mathbf{q}\left(0 ; \phi, \mathbf{p}_{0}\right) \in \mathbf{Q}_{N} D\left(\mathbf{A}^{1 / 4}\right) .
$$

(v) Finally, we consider the function that maps

$$
\mathbf{p}_{0} \in \mathbf{P}_{N} D\left(\mathbf{A}^{1 / 4}\right) \rightarrow \mathbf{q}\left(0 ; \phi, \mathbf{p}_{0}\right) \in \mathbf{Q}_{N} D\left(\mathbf{A}^{1 / 4}\right) .
$$

This function, which depends on the choice of $\phi \in H_{b, l}$, will be denoted by $\mathbf{T} \phi$; by virtue of (3.18), (3.19), and (3.20), coupled with the representation (3.17), $\mathbf{T} \phi: \mathbf{p}_{0} \rightarrow \mathbf{q}\left(0 ; \phi, \mathbf{p}_{0}\right)$ has the specific form

$$
\mathbf{T} \phi\left(\mathbf{p}_{0}\right)=-\int_{-\infty}^{0} e^{2 \mu_{1} \mathbf{A} \tau} \mathbf{Q}_{N} \mathbf{F}\left(\mathbf{p}(\tau)+\phi(\mathbf{p}(\tau)) d \tau \equiv \mathbf{q}\left(0 ; \phi, \mathbf{p}_{0}\right) .\right.
$$

VI. Once the mapping $\mathbf{T}$ has been specified, the task at hand will be

(i) to prove that for $\lambda_{N}^{1 / 2}$ and $\lambda_{N+1}^{1 / 2}-\lambda_{N}^{1 / 2}$ both sufficiently large

$$
\mathbf{T}: H_{b, l} \stackrel{\text { into }}{\rightarrow} H_{b, l}
$$

with $\mathbf{T}$ a strict contraction on $H_{b, l}$, and then

(ii) to prove that the manifold $\mathcal{M}$ defined by the graph of the (resulting) fixed point $\phi_{0}$ of $\mathbf{T}$ is an inertial manifold for the bipolar problem.

The manifold, $\mathcal{M}$, as defined above, will be a finite-dimensional Lipschitz manifold by virtue of the definition of $H_{b, l}$.

REMARK. Because it is known for the space-periodic problem that the eigenvalues of A have the form (2.14) for $n=2,3$, the requirements that both $\lambda_{N}^{1 / 2}$ and $\lambda_{N+1}^{1 / 2}-\lambda_{N}^{1 / 2}$ be sufficiently large are easy to satisfy for $\operatorname{dim} n=2$, but for $\operatorname{dim} n=3$ the spectral gap condition is only satisfied for large $\mu_{1}$.

In $\S 6$ we will establish the content of part (i) of VI, above, by proving the following result:

Theorem 6.1. Let $H_{b, l}$ be the space of Lipschitz maps $(b>0, l>0) \boldsymbol{\phi}: \mathbf{P}_{N} D\left(\mathbf{A}^{1 / 4}\right) \rightarrow$ $\mathbf{Q}_{N} D\left(\mathbf{A}^{1 / 4}\right)$ that satisfy (3.9)-(3.11). Define the mapping $\mathbf{T}$ by (3.21), where $\boldsymbol{\phi} \in H_{b, l}$, $\mathbf{p}_{0} \in \mathbf{P}_{N} D\left(\mathbf{A}^{1 / 4}\right)$ and $\mathbf{q}\left(0 ; \boldsymbol{\phi}, p_{0}\right) \in \mathbf{Q}_{N} D\left(\mathbf{A}^{1 / 4}\right)$ is the value at $t=0$ of the unique, continuous solution of $(3.18)$ (with $\mathbf{p}(t)$ the unique solution of the initial-value problem (3.13)). Then $\exists$ constants $k_{1}, k_{2}$ such that if

(i) $\lambda_{N+1}^{1 / 2}-\lambda_{N}^{1 / 2} \geq k_{1} /\left(2 \mu_{1}\right)$,

(ii) $\lambda_{N}^{1 / 2} \geq k_{2} /\left(2 \mu_{1}\right)$

then $\mathbf{T}: H_{b, l} \stackrel{\text { into }}{\rightarrow} H_{b, l}$ and is a strict contraction on $H_{b, l}$.

The proof of Theorem 6.1 depends on several lemmas, all of which are established in $\S 6$; in their order of appearance, these key lemmas read as follows: 
LEMma 6.1. For $\phi \in H_{b, l}$ we have

$$
\operatorname{supp} \mathbf{T} \boldsymbol{\phi} \subseteq\left\{\mathbf{p} \in \mathbf{P}_{N} D\left(\mathbf{A}^{1 / 4}\right)|| \mathbf{A}^{1 / 4} \mathbf{p}_{2} \mid \leq 2 r_{1}\right\}
$$

Lemma 6.2. Let $\phi \in H_{b, l}$ and $\mathbf{p}_{1}, \mathbf{p}_{2} \in \mathbf{P}_{N} D\left(\mathbf{A}^{1 / 4}\right)$. If $\mathbf{u}_{i}=\mathbf{p}_{i}+\phi\left(\mathbf{p}_{i}\right), i=1,2$, then $\exists M_{1}, M_{2}>0$ such that

$$
\begin{gathered}
\left|\mathbf{A}^{-1 / 4} \mathbf{F}\left(\mathbf{u}_{1}\right)\right| \leq M_{1} \\
\left|\mathbf{A}^{-1 / 4}\left(\mathbf{F}\left(\mathbf{u}_{1}\right)-\mathbf{F}\left(\mathbf{u}_{2}\right)\right)\right| \leq M_{2}(1+l)\left|\mathbf{A}^{1 / 4}\left(\mathbf{p}_{1}-\mathbf{p}_{2}\right)\right| .
\end{gathered}
$$

REMARK. Lemma 6.2 will be a consequence of the Lipschitz property (3.1) and the standard lemmas in Temam [15].

Lemma 6.3. If $\mathbf{p}_{0} \in \mathbf{P}_{N} D\left(\mathbf{A}^{1 / 4}\right)$, then

$$
\mathbf{T} \phi\left(\mathbf{p}_{0}\right) \in \mathbf{Q}_{N} D\left(\mathbf{A}^{1 / 4}\right) \quad \text { and } \quad\left|\mathbf{A}^{1 / 4}\left[\mathbf{T} \phi\left(\mathbf{p}_{0}\right)\right]\right| \leq b^{\prime}
$$

with $b^{\prime}=e^{-1 / 2} \mu_{1}^{-1} \lambda_{N+1}^{-1 / 2}<b$ (for $\lambda_{N+1}^{1 / 2}$ sufficiently large).

REMARK. Lemma 6.3 will be a direct consequence of the explicit representation of T $\boldsymbol{\phi}$, i.e., (3.21), with $\phi \in H_{b . l}$.

LEMma 6.4. Assume that

$$
\sigma_{N}=2 \mu_{1}\left(\lambda_{N+1}-\lambda_{N}\right)-M_{2}(1+l) \lambda_{N}^{1 / 2}>0
$$

Then for $\phi \in H_{b, l}$, and $\mathbf{p}_{01}, \mathbf{p}_{02} \in \mathbf{P}_{N} D\left(\mathbf{A}^{1 / 4}\right)$, we have

$$
\left|\mathbf{A}^{1 / 4}\left(\mathbf{T} \phi\left(\mathbf{p}_{01}\right)-\mathbf{T} \phi\left(\mathbf{p}_{02}\right)\right)\right| \leq l^{\prime}\left|\mathbf{A}^{1 / 4}\left(\mathbf{p}_{01}-\mathbf{p}_{02}\right)\right|
$$

where

$$
\begin{gathered}
l^{\prime}=M_{2}(1+l) \lambda_{N+1}^{-1 / 2}\left[\left(2 \mu_{1}\right)^{-1}+\left(2 \mu_{1}-r_{N} \xi_{N}\right)^{-1}\right] e^{-1 / 2} \exp \left(\frac{r_{N} \xi_{N}}{4 \mu_{1}}\right), \\
r_{N}=\lambda_{N} / \lambda_{N+1} \\
\xi_{N}=2 \mu_{1}+M_{2}(1+l) \lambda_{N}^{-1 / 2} .
\end{gathered}
$$

REMARK. As a consequence of (3.26), $\mathbf{T} \phi \in H_{b . l^{\prime}}$. It is proven in Lemma 6.6 that the $k_{i}, i=1,2$, in the statement of Theorem 6.1 may be chosen so that when (i) and (ii) of Theorem 6.1 are satisfied, so is (3.25).

LEMmA 6.5. Assume, again, that $\sigma_{N}>0$, where $\sigma_{N}$ is defined by (3.25). Then for $\phi_{1}, \phi_{2} \in H_{b, l}$ and $\mathbf{p}_{0} \in \mathbf{P}_{N} D\left(\mathbf{A}^{1 / 4}\right)$ we have

$$
\left|\mathbf{A}^{1 / 4}\left(\mathbf{T} \phi_{1}\left(\mathbf{p}_{0}\right)-\mathbf{T} \phi_{2}\left(\mathbf{p}_{0}\right)\right)\right| \leq L\left\|\phi_{1}-\phi_{2}\right\|
$$

with $L=\frac{M_{2}}{2 \mu_{1}}\left(2 e^{-1 / 2} \lambda_{N+1}^{-1 / 2}-\lambda_{N}^{-1 / 2} l^{\prime}\right)$ and $\left\|\phi_{1}-\phi_{2}\right\|$ given by $(3.11)$.

The final lemma in this series actually serves to establish Theorem 6.1, i.e., 
Lemma 6.6. For $0<l<1$, if (i) and (ii) of Theorem 6.1 hold with

$$
k_{1}=2 M_{2}(1+l) l^{-1}, \quad k_{2}=2 M_{2}\left(2 e^{-1 / 2}+l\right)
$$

then $\sigma_{N}>0$ (with $\sigma_{N}$ as defined by $(3.25)$ ), $l^{\prime}$ as given by $\left(3.27 \mathrm{a}, \mathrm{b}, \mathrm{c}\right.$ ) satisfies $l^{\prime}<l$ (so that, by virtue of (3.26), $\mathbf{T} \phi \in H_{b, l}$ ), and $L \leq \frac{1}{2}$ (so that, in view of (3.28), $\mathbf{T}$ is a strict contraction on $\left.H_{b, l}\right)$.

The content of part (ii) of VI, above, is established in $\S 7$. In fact, to show that the graph $\mathcal{M}$ of the fixed point $\phi_{0}$ of $\mathbf{T}$ is an inertial manifold we essentially need to prove only that $\mathcal{M}$ attracts exponentially all orbits of $S_{\mu_{1}}(t)$; as was previously indicated, the fact that $\mathcal{M} \subseteq D\left(\mathbf{A}^{1 / 4}\right)$ is a finite-dimensional Lipschitz manifold follows directly from the definition of $H_{b, l}$ and the invariance of $\mathcal{M}$ under $S_{\mu_{1}}(t), \forall t \geq 0$, and is relatively easy to establish. In addition, the fact that $\mathcal{M}$ attracts exponentially all orbits of the evolution equation (2.18) will follow once we prove, in $\S 7$, that $\mathcal{M}$ attracts exponentially all orbits of the modified equation (3.7), and this requires a squeezing property for the modified equation (3.7); from this squeezing property we will obtain, in $\S 7$, the following result:

TheOREM 7.1. Let $\mathcal{M}$ be the graph of the fixed point $\phi_{0}$ of $\mathbf{T}$, whose existence is implied by Theorem 6.1 and the completeness of the space $H_{b, l}$ with respect to the norm specified in (3.11). Then $\exists t_{0}>0$ such that for $\mathbf{u}_{0} \in D\left(\mathbf{A}^{1 / 4}\right)$ and $t \leq t_{0}$

$$
\operatorname{dist}\left(S_{\mu_{1}}(t) \mathbf{u}_{0}, \mathcal{M}\right) \leq \exp \left(-\frac{t}{2 t_{0}} \ln 2\right) \operatorname{dist}\left(\mathbf{u}_{0}, \mathcal{M}\right)
$$

In the next section we will begin working our way through the program described in steps I-VI by establishing the Lipschitz property (3.1) for solutions of the initial-value problem (2.18), (2.19).

4. The Lipschitz property. The purpose of this section is to prove the following:

Lemma 4.1. Let $\mathbf{R}(\mathbf{u})$ be defined by (2.15)-(2.17) with $\mathbf{e}=\mathbf{e}(\mathbf{u})$. Then $\mathbf{R}(\mathbf{u})$ is a Lipschitz function on the bounded sets of $D\left(\mathbf{A}^{1 / 4}\right)$ with values in $D\left(\mathbf{A}^{-1 / 4}\right)$, i.e., for $M>0, \exists C_{M}>0$ such that (3.1) holds $\forall \mathbf{u}, \mathbf{v} \in D\left(\mathbf{A}^{1 / 4}\right)$ with $\left|\mathbf{A}^{1 / 4} \mathbf{u}\right| \leq M$ and $\left|\mathbf{A}^{1 / 4} \mathbf{v}\right| \leq M$.

Proof. Let $\mathbf{u}, \mathbf{v} \in D\left(\mathbf{A}^{1 / 4}\right)$ and set $\mathbf{w}=\mathbf{u}-\mathbf{v}$. With $\mathbf{A}_{p}$ defined as per (2.15) we have

$$
\begin{aligned}
& \left(\mathbf{A}_{p}(\mathbf{u})-\mathbf{A}_{p}(\mathbf{v}), \mathbf{w}\right)=\int_{\Omega}\left[\mathbf{A}_{p}(\mathbf{u})_{i}-\mathbf{A}_{p}(\mathbf{v})_{i}\right] w_{i} d \mathbf{x} \\
& \quad=\int_{\Omega}\left\{\frac{\partial}{\partial x_{j}}\left[\left(\epsilon+|\mathbf{e}(\mathbf{u})|^{2}\right)^{\frac{p-2}{2}} e_{i j}(\mathbf{u})\right]-\frac{\partial}{\partial x_{j}}\left[\left(\epsilon+|\mathbf{e}(\mathbf{v})|^{2}\right)^{\frac{p-2}{2}} e_{i j}(\mathbf{v})\right]\right\} w_{i} d \mathbf{x} .
\end{aligned}
$$

Integrating (4.1) by parts, and using the fact that, as a consequence of the space periodicity satisfied by $\mathbf{u}$ and $\mathbf{v}$,

$$
\int_{\partial \Omega}\left[\left(\epsilon+|\mathbf{e}(\mathbf{u})|^{2}\right)^{\frac{p-2}{2}} e_{i j}(u)-\left(\epsilon+|\mathbf{e}(\mathbf{v})|^{2}\right)^{\frac{p-2}{2}} e_{i j}(\mathbf{v})\right] w_{i} \nu_{j} d S=0,
$$


we easily find that

$$
\begin{aligned}
\left(\mathbf{A}_{p}(\mathbf{u})\right. & \left.-\mathbf{A}_{p}(\mathbf{v}), \mathbf{w}\right) \\
\quad= & -\int_{\Omega}\left[\left(\epsilon+|\mathbf{e}(\mathbf{u})|^{2}\right)^{\frac{p-2}{2}} e_{i j}(\mathbf{u})-\left(\epsilon+|\mathbf{e}(\mathbf{v})|^{2}\right)^{\frac{p-2}{2}} e_{i j}(\mathbf{v})\right] \frac{\partial w_{i}}{\partial x_{j}} d \mathbf{x} .
\end{aligned}
$$

We now set

$$
\begin{gathered}
r_{p}(\mathbf{e})=\frac{1}{p}\left(\epsilon+|\mathbf{e}|^{2}\right)^{p / 2}, \\
\tilde{e}_{i j}(t)=e_{i j}(\mathbf{u})+t\left(e_{i j}(\mathbf{v})-e_{i j}(\mathbf{u})\right), \quad 0 \leq t \leq 1 .
\end{gathered}
$$

Then

$$
\frac{\partial r_{p}}{\partial e_{i j}}=\left(\epsilon+|\mathbf{e}|^{2}\right)^{\frac{p-2}{2}} e_{i j}
$$

so that

$$
\begin{gathered}
{\left[\left(\epsilon+\left.\mathbf{e}(\mathbf{u})\right|^{2}\right)^{\frac{p-2}{2}} e_{i j}(\mathbf{u})-\left(\epsilon+|\mathbf{e}(\mathbf{v})|^{2}\right)^{\frac{p-2}{2}} e_{i j}(\mathbf{v})\right]} \\
=\int_{0}^{1} \frac{\partial}{\partial t}\left(\frac{\partial r_{p}}{\partial e_{i j}}\left(\tilde{e}_{i j}(t)\right)\right) d t
\end{gathered}
$$

Employing (4.7) in (4.3) yields

$$
\begin{aligned}
& \left|\left(\mathbf{A}_{p}(\mathbf{u})-\mathbf{A}_{p}(\mathbf{v}), \mathbf{w}\right)\right| \\
& \quad=\left|\int_{\Omega}\left\{\int_{0}^{1} \frac{\partial}{\partial t}\left(\frac{\partial r_{p}}{\partial e_{i j}}\left(\tilde{e}_{i j}(t)\right)\right) d t\right\} \frac{\partial w_{i}}{\partial x_{j}} d \mathbf{x}\right| \\
& \leq \int_{\Omega} \int_{0}^{1}\left|\frac{\partial}{\partial t}\left(\frac{\partial r_{p}}{\partial e_{i j}}\left(\tilde{e}_{i j}(t)\right)\right) \frac{\partial w_{i}}{\partial x_{j}}\right| d t d \mathbf{x} .
\end{aligned}
$$

However, by virtue of (4.4)-(4.6)

$$
\begin{aligned}
\frac{\partial^{2} r_{p}}{\partial t \partial e_{i j}}\left(\tilde{e}_{i j}(t)\right) & =\frac{\partial^{2} r_{p}}{\partial e_{i j} \partial e_{k l}}\left(\tilde{e}_{i j}(t)\right) \frac{\partial \tilde{e}_{k l}}{\partial t} \\
& =\frac{\partial^{2} r_{p}}{\partial e_{i j} \partial e_{k l}}\left(\tilde{e}_{i j}(t)\right)\left(e_{k l}(\mathbf{v})-e_{k l}(\mathbf{u})\right)
\end{aligned}
$$

while

$$
\frac{\partial^{2} r_{p}}{\partial e_{i j} \partial e_{k l}}=\frac{p-2}{2}\left(\epsilon+|\mathbf{e}|^{2}\right)^{\frac{p-4}{2}} e_{i j} e_{k l}+\left(\epsilon+|\mathbf{e}|^{2}\right)^{\frac{p-2}{2}} \delta_{i j} \delta_{j l}
$$

Therefore, for any $\boldsymbol{\xi} \neq 0, \boldsymbol{\eta} \neq 0$

$$
\begin{aligned}
& \left|\frac{\partial^{2} r_{p}}{\partial e_{i j} \partial e_{k l}} \xi_{i j} \eta_{k l}\right| \\
& \quad=\left|\frac{p-2}{2}\left(\epsilon+|\mathbf{e}|^{2}\right)^{\frac{p-4}{2}} e_{i j} e_{k l} \xi_{i j} \eta_{k l}+\left(\epsilon+|\mathbf{e}|^{2}\right)^{\frac{p-2}{2}} \xi_{k j} \eta_{k j}\right| \\
& \quad \leq\left|\frac{p-2}{2}\right|\left(\epsilon+|\mathbf{e}|^{2}\right)^{\frac{p-4}{2}}\left|e_{i j} \xi_{i j}\right|\left|e_{k l} \eta_{k l}\right|+\left(\epsilon+|\mathbf{e}|^{2}\right)^{\frac{p-2}{2}}\left|\xi_{k j} \eta_{k j}\right| \\
& \quad \leq\left|\frac{p-2}{2}\right|\left(\epsilon+|\mathbf{e}|^{2}\right)^{\frac{p-4}{2}}|\mathbf{e}|^{2}|\boldsymbol{\xi}||\boldsymbol{\eta}|+\left(\epsilon+|\mathbf{e}|^{2}\right)^{\frac{p-2}{2}}|\boldsymbol{\xi}||\boldsymbol{\eta}| \\
& \quad \leq\left(\frac{2-p}{2}+1\right) \varepsilon^{\frac{p-2}{2}}|\boldsymbol{\xi}||\boldsymbol{\eta}|,
\end{aligned}
$$


because $0<p \leq 2$. Combining (4.8)-(4.11), we obtain

$$
\begin{aligned}
& \left|\left(\mathbf{A}_{p}(\mathbf{u})-\mathbf{A}_{p}(\mathbf{v}), \mathbf{w}\right)\right| \\
& \quad \leq \int_{\Omega} \int_{0}^{1}\left|\frac{\partial^{2} r_{p}}{\partial e_{i j} \partial e_{k l}}\left(\tilde{e}_{i j}(t)\right)\left(e_{k l}(\mathbf{v})-e_{k l}(\mathbf{u})\right) \cdot \frac{\partial w_{i}}{\partial x_{j}}\right| d t d \mathbf{x} \\
& \quad \leq\left(\frac{2-p}{2}+1\right) \epsilon^{\frac{p-2}{2}} \int_{\Omega} \int_{0}^{1}\left(\sum_{k, l}\left|e_{k l}(\mathbf{v}-\mathbf{u})\right|^{2}\right)^{1 / 2}\left(\sum_{i, j}\left|\frac{\partial w_{i}}{\partial x_{j}}\right|^{2}\right)^{1 / 2} d t d \mathbf{x} \\
& \quad \leq\left(\frac{2-p}{2}+1\right) \epsilon^{\frac{p-2}{2}} \int_{\Omega}\left(\sum_{k, l}\left|e_{k l}(\mathbf{v}-\mathbf{u})\right|^{2}\right)^{1 / 2}\left(\sum_{i, j}\left|\frac{\partial w_{i}}{\partial x_{j}}\right|^{2}\right)^{1 / 2} d \mathbf{x}
\end{aligned}
$$

or, for some $C_{1}=C_{1}(\Omega)>0$

$$
\begin{aligned}
& \left|\left(\mathbf{A}_{p}(\mathbf{u})-\mathbf{A}_{p}(\mathbf{v}), \mathbf{w}\right)\right| \\
& \quad \leq\left(\frac{2-p}{2}+1\right) \epsilon^{\frac{p-2}{2}} C_{1}\|\mathbf{u}-\mathbf{v}\|_{H^{1}(\Omega)}\|\mathbf{w}\|_{H^{1}(\Omega)} .
\end{aligned}
$$

Next, consider $\mathbf{B}(\mathbf{u}, \mathbf{v})$ as defined by $(2.16)$. We have

$$
\begin{aligned}
|(\mathbf{B}(\mathbf{u}, \mathbf{u})-\mathbf{B}(\mathbf{v}, \mathbf{v}), \mathbf{w})| & \left|\int_{\Omega}\left(u_{j} \frac{\partial u_{i}}{\partial x_{j}}-v_{j} \frac{\partial v_{i}}{\partial x_{j}}-v_{j} \frac{\partial v_{i}}{\partial x_{j}}\right) w_{i} d \mathbf{x}\right| \\
\leq & \left|\int_{\Omega}\left(u_{j}-v_{j}\right) \frac{\partial u_{i}}{\partial x_{j}} w_{i} d \mathbf{x}\right|+\left|\int_{\Omega} v_{i} \frac{\partial}{\partial x_{j}}\left(u_{i}-v_{i}\right) w_{i} d \mathbf{x}\right| \\
\leq & \left(\int_{\Omega}\left(u_{j}-v_{j}\right)\left(u_{j}-v_{j}\right) w_{i} w_{i} d \mathbf{x}\right)^{1 / 2}\left(\int_{\Omega} \frac{\partial u_{i}}{\partial x_{j}} \frac{\partial u_{i}}{\partial x_{j}} d \mathbf{x}\right)^{1 / 2} \\
& +\left(\int_{\Omega} v_{j} v_{j} w_{i} w_{i} d \mathbf{x}\right)^{1 / 2}\left(\int_{\Omega} \sum_{i, j}\left|\frac{\partial\left(u_{i}-v_{i}\right)}{\partial x_{j}}\right|^{2} d \mathbf{x}\right)^{1 / 2}
\end{aligned}
$$

Using the Sobolev embedding $H^{1}(\Omega) \hookrightarrow L^{4}(\Omega)$, which is valid for $\Omega=[0, L]^{n}, n=2,3$, we obtain from this last estimate

$$
\begin{aligned}
|(\mathbf{B}(\mathbf{u}, \mathbf{u})-\mathbf{B}(\mathbf{v}, \mathbf{v}), \mathbf{w})| & \left(\int_{\Omega} \sum_{j}\left(u_{j}-v_{j}\right)^{4} d \mathbf{x}\right)^{1 / 4}\left(\int_{\Omega} \sum_{i, j}\left|\frac{\partial u_{i}}{\partial x_{j}}\right|^{2} d \mathbf{x}\right)^{1 / 2}\left(\int_{\Omega} \sum_{i}\left|w_{i}\right|^{4} d \mathbf{x}\right)^{1 / 4} \\
& +\left(\int_{\Omega} \sum_{j}\left|v_{j}\right|^{4} d \mathbf{x}\right)^{1 / 4}\left(\int_{\Omega} \sum_{i, j}\left|\frac{\partial\left(u_{i}-v_{i}\right)}{\partial x_{j}}\right|^{2} d \mathbf{x}\right)^{1 / 2}\left(\int_{\Omega} \sum_{i}\left|w_{i}\right|^{4} d \mathbf{x}\right)^{1 / 4},
\end{aligned}
$$

so that for some $C_{2}=C_{2}(\Omega)>0$

$$
\begin{aligned}
& |(\mathbf{B}(\mathbf{u}, \mathbf{u})-\mathbf{B}(\mathbf{v}, \mathbf{v}), \mathbf{w})| \\
& \quad \leq C_{2}\left(\|\mathbf{u}\|_{H^{1}(\Omega)}+\|\mathbf{v}\|_{H^{1}(\Omega)}\right)\|\mathbf{u}-\mathbf{v}\|_{H^{1}(\Omega)}\|\mathbf{w}\|_{H^{1}(\Omega)} .
\end{aligned}
$$


Now, by virtue of the definition of $\mathbf{R}(\mathbf{u})$, i.e. (2.15)-(2.17),

$$
\mathbf{R}(\mathbf{u})-\mathbf{R}(\mathbf{v})=-2 \mu_{0}\left(\mathbf{A}_{p}(\mathbf{u})-\mathbf{A}_{p}(\mathbf{v})\right)+(\mathbf{B}(\mathbf{u}, \mathbf{u})-\mathbf{B}(\mathbf{v}, \mathbf{v}))
$$

so, combining (4.17) with (4.13) and (4.16), we find that

$$
\begin{aligned}
\mid(\mathbf{R}(\mathbf{u}) & -\mathbf{R}(\mathbf{v}), \mathbf{w}) \mid \\
\leq & 2 \mu_{0}\left|\left(\mathbf{A}_{p}(\mathbf{u})-\mathbf{A}_{p}(\mathbf{v}), \mathbf{w}\right)\right|+|(\mathbf{B}(\mathbf{u}, \mathbf{u})-\mathbf{B}(\mathbf{v}, \mathbf{v}), \mathbf{w})| \\
\leq & {\left[2 \mu_{0}\left(\frac{2-p}{2}+1\right) \epsilon^{\frac{p-2}{2}} C_{1}+C_{2}\left(\|\mathbf{u}\|_{H^{1}(\Omega)}+\|\mathbf{v}\|_{H^{1}(\Omega)}\right)\right]\|\mathbf{u}-\mathbf{v}\|_{H^{1}(\Omega)}\|\mathbf{w}\|_{H^{1}(\Omega)} . }
\end{aligned}
$$

Employing (2.12c), i.e., the equivalence between the norms $\|\mathbf{u}\|_{H^{1}(\Omega)}$ and $\left|\mathbf{A}^{1 / 4} \mathbf{u}\right|$ for $\mathbf{u} \in D\left(\mathbf{A}^{1 / 4}\right),(4.18)$ yields the following estimate, for some $C_{3}=C_{3}(\Omega)>0$ :

$$
\begin{aligned}
\mid(\mathbf{R}(\mathbf{u}) & -\mathbf{R}(\mathbf{v}), \mathbf{w}) \mid \\
\leq & C_{3}\left[2 \mu_{0}\left(\frac{2-p}{2}+1\right) \epsilon^{\frac{p-2}{2}} C_{1}+C_{2}\left(\left|\mathbf{A}^{1 / 4} \mathbf{u}\right|+\left|\mathbf{A}^{1 / 4} \mathbf{v}\right|\right)\right] \\
& \times\left|\mathbf{A}^{1 / 4}(\mathbf{u}-\mathbf{v})\right|\left|\mathbf{A}^{1 / 4} \mathbf{w}\right| .
\end{aligned}
$$

Thus, for $\mathbf{u}, \mathbf{v} \in D\left(\mathbf{A}^{1 / 4}\right)$ satisfying $\left|\mathbf{A}^{1 / 4} \mathbf{u}\right| \leq M,\left|A^{1 / 4} \mathbf{v}\right| \leq M$, for some $M>0$,

$$
|(\mathbf{R}(\mathbf{u})-\mathbf{R}(\mathbf{v}), \mathbf{w})| \leq C_{M}\left|\mathbf{A}^{1 / 4}(\mathbf{u}-\mathbf{v})\right|\left|\mathbf{A}^{1 / 4} \mathbf{w}\right|
$$

with

$$
C_{M}=C_{3}\left[2 \mu_{0}\left(\frac{2-p}{2}+1\right) \epsilon^{\frac{p-2}{2}} C_{1}+C_{2} M\right] .
$$

The required estimate, i.e., (3.1), is now a direct consequence of (4.20).

5. The squeezing property. Our goal in this section is to prove the following squeezing property for orbits of the nonlinear semigroup $S_{\mu_{1}}$ :

TheOREm 5.1. Let $\mathbf{w}_{1}, \ldots, \mathbf{w}_{N}$ be the first $N$ eigenfunctions of the operator $\mathbf{A}$ and $\mathbf{P}_{N}: H_{\text {per }} \rightarrow \operatorname{span}\left\{\mathbf{w}_{1}, \ldots, \mathbf{w}_{N}\right\}$ the projective operator; set $\mathbf{Q}_{N}=\mathbf{I}-\mathbf{P}_{N}$, where $\mathbf{I}$ is the identity map on $H_{\text {per. }}$. Let $\gamma>0$ be given. Then for $\mathbf{u}, \mathbf{v} \in D\left(\mathbf{A}^{1 / 4}\right)$ satisfying (3.3), for $t \in[0, T], T>0$, and with $M>0$ as in the statement of the Lipschitz property (3.1), $\exists c_{i}>0, i=1,2$, depending only on $\gamma, M, T, \mathbf{f}, \mu_{0}, \mu_{1}, \epsilon$, and $\Omega$, such that for every $N$ and every $t \in[0, T]$ either (3.4) holds or (3.5) does. In addition,

$$
\left|\mathbf{A}^{-1 / 4}(\mathbf{u}(t)-\mathbf{v}(t))\right| \leq \exp \left(\frac{C_{\Lambda I}^{2}}{\mu_{1}} t\right)\left|\mathbf{A}^{-1 / 4}(\mathbf{u}(0)-\mathbf{v}(0))\right| .
$$

The proof of Theorem 5.1 hinges on a key lemma which we will establish first. Consider two different solutions $\mathbf{u}$ and $\mathbf{v}$ of the initial-value problem (2.18), (2.19) corresponding to the initial values $\mathbf{u}_{0}, \mathbf{v}_{0} \in D\left(\mathbf{A}^{1 / 4}\right)$, where $\mathbf{f} \in L^{2}\left(Q_{T}\right), Q_{T}=\Omega \times[0, T)$. We set $\mathbf{w}(t)=\mathbf{u}(t)-\mathbf{v}(t)$ and study the behavior of the quotient

$$
q(t)=\left|\mathbf{A}^{1 / 4} \mathbf{w}(t)\right|^{2} /\left|\mathbf{A}^{-1 / 4} \mathbf{w}(t)\right|^{2}
$$


LEMma 5.1. Let $\mathbf{u}, \mathbf{v} \in D\left(\mathbf{A}^{1 / 4}\right)$ be the unique solutions of the initial-value problems

$$
\begin{gathered}
\left\{\begin{array}{c}
\frac{d \mathbf{u}}{d t}+2 \mu_{1} \mathbf{A} \mathbf{u}+\mathbf{R}(\mathbf{u})=\mathbf{0} \\
\mathbf{u}(0)=\mathbf{u}_{0}
\end{array}\right. \\
\left\{\begin{array}{c}
\frac{d \mathbf{v}}{d t}+2 \mu_{1} \mathbf{A} \mathbf{v}+\mathbf{R}(\mathbf{v})=0 \\
\mathbf{v}(0)=\mathbf{v}_{0}
\end{array}\right.
\end{gathered}
$$

Let $M>0$ and suppose that $\forall t \in[0, T],\left|\mathbf{A}^{1 / 4} \mathbf{u}(t)\right| \leq M,\left|\mathbf{A}^{1 / 4} \mathbf{v}(t)\right| \leq M$. Then $\exists c_{3}>0$ such that $\mathbf{w}(t)=\mathbf{u}(t)-\mathbf{v}(t)$ satisfies

$$
\frac{\left|\mathbf{A}^{1 / 4} \mathbf{w}(\tau)\right|^{2}}{\left|\mathbf{A}^{-1 / 4} \mathbf{w}(t)\right|^{2}} \leq \frac{\left|\mathbf{A}^{1 / 4} \mathbf{w}(t)\right|^{2}}{\left|\mathbf{A}^{-1 / 4} \mathbf{w}(t)\right|^{2}} \exp \left(c_{3}(\tau-t)\right)
$$

for $0<t<\tau<T$.

Proof of Lemma 5.1. For $t \in[0, T]$ we define the quotient $q(t)$ as per (5.2), i.e.,

$$
q(t)=\frac{\left(\mathbf{A}^{1 / 4} \mathbf{w}, \mathbf{A}^{1 / 4} \mathbf{w}\right)}{\left(A^{-1 / 4} \mathbf{w}, \mathbf{A}^{-1 / 4} \mathbf{w}\right)} .
$$

Differentiating $q(t)$, we obtain $\left({ }^{\prime}=d / d t\right)$

$$
\begin{array}{r}
\frac{d q}{d t}=\frac{2}{\left(\mathbf{A}^{-1 / 4} \mathbf{w}, \mathbf{A}^{-1 / 4} \mathbf{w}\right)^{2}}\left[\left(\mathbf{A}^{-1 / 4} \mathbf{w}, \mathbf{A}^{-1 / 4} \mathbf{w}\right)\left(\mathbf{A}^{1 / 4} \mathbf{w}^{\prime}, \mathbf{A}^{1 / 4} \mathbf{w}\right)\right. \\
\left.-\left(\mathbf{A}^{-1 / 4} \mathbf{w}, \mathbf{A}^{-1 / 4} \mathbf{w}\right)\left(\mathbf{A}^{1 / 4} \mathbf{w}, \mathbf{A}^{1 / 4} \mathbf{w}\right)\right]
\end{array}
$$

or

$$
\frac{d q}{d t}=\frac{2}{\left|\mathbf{A}^{-1 / 4} \mathbf{w}(t)\right|^{2}}\left[\left(\mathbf{w}^{\prime}, \mathbf{A}^{1 / 2} \mathbf{w}\right)-q(t)\left(\mathbf{w}, \mathbf{A}^{-1 / 2} \mathbf{w}\right)\right]
$$

However,

$$
\frac{d \mathbf{w}}{d t}+2 \mu_{1} \mathbf{A}(\mathbf{w})+\mathbf{R}(\mathbf{u})-\mathbf{R}(\mathbf{v})=\mathbf{0}
$$

so

$$
\frac{d q}{d t}=\frac{-2}{\left|\mathbf{A}^{-1 / 4} \mathbf{w}\right|^{2}}\left(2 \mu_{1} \mathbf{A} \mathbf{w}+\mathbf{R}(\mathbf{u}), \mathbf{A}^{1 / 2} \mathbf{w}-q(t) \mathbf{A}^{-1 / 2} \mathbf{w}\right),
$$

from which it follows that

$$
\frac{d q}{d t}=\frac{-2}{\left|\mathbf{A}^{-1 / 4} \mathbf{w}\right|^{2}}\left(2 \mu_{1} \mathbf{A}^{3 / 4} \mathbf{w}+\mathbf{A}^{-1 / 4}(\mathbf{R}(\mathbf{u})-\mathbf{R}(\mathbf{v})), \mathbf{A}^{3 / 4} \mathbf{w}-q(t) \mathbf{A}^{-1 / 4} \mathbf{w}\right) .
$$

If we now make note of the fact that

$$
\begin{aligned}
\left(q \mathbf{A}^{-1 / 4} \mathbf{w}, \mathbf{A}^{3 / 4} \mathbf{w}-q \mathbf{A}^{-1 / 4} \mathbf{w}\right) \\
\quad=q\left(\mathbf{A}^{-1 / 4} \mathbf{w}, \mathbf{A}^{3 / 4} \mathbf{w}\right)-q^{2}\left(\mathbf{A}^{-1 / 4} \mathbf{w}, \mathbf{A}^{-1 / 4} \mathbf{w}\right) \\
\quad=q\left(\mathbf{A}^{1 / 4} \mathbf{w}, \mathbf{A}^{1 / 4} \mathbf{w}\right)-q^{2}\left(\mathbf{A}^{-1 / 4} \mathbf{w}, \mathbf{A}^{-1 / 4} \mathbf{w}\right) \\
\quad \equiv 0
\end{aligned}
$$


by virtue of the definition of $q(t)$, i.e., (5.2), then

$$
\begin{aligned}
\left(\mathbf{A}^{3 / 4} \mathbf{w}, \mathbf{A}^{3 / 4} \mathbf{w}-q \mathbf{A}^{-1 / 4} \mathbf{w}\right) \\
\quad=\left(\mathbf{A}^{3 / 4} \mathbf{w}-q \mathbf{A}^{-1 / 4} \mathbf{w}, \mathbf{A}^{3 / 4} \mathbf{w}-q \mathbf{A}^{-1 / 4} \mathbf{w}\right) \\
\quad=\left|\mathbf{A}^{3 / 4} \mathbf{w}-q \mathbf{A}^{-1 / 4} \mathbf{w}\right|^{2}
\end{aligned}
$$

Employing (5.10) in (5.9) and using the Cauchy-Schwarz inequality, we find that

$$
\begin{aligned}
\frac{d q}{d t}+ & \frac{4 \mu_{1}}{\left|\mathbf{A}^{-1 / 4} \mathbf{w}\right|^{2}}\left|\mathbf{A}^{3 / 4} \mathbf{w}-q \mathbf{A}^{-1 / 4} \mathbf{w}\right|^{2} \\
& =\frac{-2}{\left|\mathbf{A}^{-1 / 4} \mathbf{w}\right|^{2}}\left(\mathbf{A}^{-1 / 4}(\mathbf{R}(\mathbf{u})-\mathbf{R}(\mathbf{v})), \mathbf{A}^{3 / 4} \mathbf{w}-q \mathbf{A}^{-1 / 4} \mathbf{w}\right) \\
& \leq \frac{2}{\left|\mathbf{A}^{-1 / 4} \mathbf{w}\right|^{2}}\left|\mathbf{A}^{-1 / 4}(\mathbf{R}(\mathbf{u})-\mathbf{R}(\mathbf{v}))\right| \cdot\left|\mathbf{A}^{3 / 4} \mathbf{w}-q \mathbf{A}^{-1 / 4} \mathbf{w}\right| \\
& \leq \frac{4 \mu_{1}}{\left|A^{-1 / 4} \mathbf{w}\right|^{2}}\left|\mathbf{A}^{3 / 4} \mathbf{w}-q \mathbf{A}^{-1 / 4}\right|^{2}+\frac{1}{\mu_{1}} \frac{\left|A^{-1 / 4}(\mathbf{R}(\mathbf{u})-\mathbf{R}(\mathbf{v}))\right|^{2}}{\left|\mathbf{A}^{-1 / 4} \mathbf{w}\right|^{2}}
\end{aligned}
$$

where for the last estimate we have used the arithmetic-geometric mean inequality. We now avail ourselves of the Lipschitz property (3.1), which is valid for $\mathbf{u}, \mathbf{v} \in D\left(\mathbf{A}^{1 / 4}\right)$ satisfying $\left|\mathbf{A}^{1 / 4} \mathbf{u}\right| \leq M,\left|\mathbf{A}^{1 / 4} \mathbf{v}\right| \leq M$ for $M>0$; employing (3.1) in the last estimate in (5.11), and making use of the definition (5.2) of $q(t)$, we find that

$$
\frac{d q}{d t} \leq \frac{1}{\mu_{1}} \cdot C_{M}^{2} q
$$

where $C_{M}$ is given by (4.21). Integration of (5.12) from $\tau$ to $t$ yields (5.5) with $c_{3}=$ $\frac{1}{\mu_{2}} C_{M}^{2}$. The lemma is proved.

We are now in a position to establish the squeezing property for $S_{\mu_{1}}$ :

Proof of Theorem 5.1. We begin by taking the scalar product in $L^{2}$ of (5.8) with $\mathbf{A}^{-1 / 2} \mathbf{w}$; using the Cauchy-Schwarz inequality and the Lipschitz property (3.1), we obtain

$$
\begin{aligned}
\frac{1}{2} \frac{d}{d t} \mid & \left.\mathbf{A}^{-1 / 4} \mathbf{w}\right|^{2}+2 \mu_{1}\left|\mathbf{A}^{1 / 4} \mathbf{w}\right|^{2} \\
& =-\left(\mathbf{A}^{-1 / 4}(\mathbf{R}(\mathbf{u})-\mathbf{R}(\mathbf{v})), \mathbf{A}^{-1 / 4} \mathbf{w}\right) \\
& \leq\left|\mathbf{A}^{-1 / 4}(\mathbf{R}(\mathbf{u})-\mathbf{R}(\mathbf{v}))\right| \cdot\left|\mathbf{A}^{-1 / 4} \mathbf{w}\right| \\
& \leq C_{M}\left|\mathbf{A}^{1 / 4} \mathbf{w}\right|\left|\mathbf{A}^{-1 / 4} \mathbf{w}\right|
\end{aligned}
$$

with $C_{M}$ given by (4.21). Employing the arithmetic-geometric mean inequality, we are led from (5.13) to the estimate

$$
\frac{d}{d t}\left|\mathbf{A}^{-1 / 4} \mathbf{w}\right|^{2}+4 \mu_{1}\left|A^{1 / 4} \mathbf{w}\right|^{2} \leq 3 \mu_{1}\left|\mathbf{A}^{1 / 4} \mathbf{w}\right|^{2}+\frac{4 C_{M}^{2}}{3 \mu_{1}}\left|\mathbf{A}^{-1 / 4} \mathbf{w}\right|^{2},
$$

from which it follows that

$$
\frac{d}{d t}\left|\mathbf{A}^{-1 / 4} \mathbf{w}\right|^{2}+\left|\mathbf{A}^{-1 / 4} \mathbf{w}\right|^{2}\left(\frac{\mu_{1}\left|\mathbf{A}^{1 / 4} \mathbf{w}\right|^{2}}{\left|\mathbf{A}^{-1 / 4} \mathbf{w}\right|^{2}}-\frac{4 C_{M I}^{2}}{3 \mu_{1}}\right) \leq 0 .
$$


By virtue of Lemma 5.1, however, for $0<t<t_{0}<T$ we have

$$
\frac{\left|\mathbf{A}^{1 / 4} \mathbf{w}(t)\right|^{2}}{\left|\mathbf{A}^{-1 / 4} \mathbf{w}(t)\right|^{2}} \geq \frac{\left|\mathbf{A}^{1 / 4} \mathbf{w}\left(t_{0}\right)\right|^{2}}{\left|\mathbf{A}^{-1 / 4} \mathbf{w}\left(t_{0}\right)\right|^{2}} \exp \left(-c_{3}\left(t_{0}-t\right)\right) \geq \eta \exp \left(-c_{3} t_{0}\right)
$$

with

$$
\eta=\left|\mathbf{A}^{1 / 4} \mathbf{w}\left(t_{0}\right)\right|^{2} /\left|\mathbf{A}^{-1 / 4} \mathbf{w}\left(t_{0}\right)\right|^{2} .
$$

Combining (5.15) and (5.16), we find that

$$
\frac{d}{d t}\left|\mathbf{A}^{-1 / 4} \mathbf{w}(t)\right|^{2}+\left|\mathbf{A}^{-1 / 4} \mathbf{w}(t)\right|^{2}\left(\mu_{1} \eta \exp \left(-c_{3} t_{0}\right)-\frac{4 C_{M}^{2}}{3 \mu_{1}}\right) \leq 0
$$

so that upon integrating from zero to $t_{0}$ we obtain

$$
\left|\mathbf{A}^{-1 / 4} \mathbf{w}\left(t_{0}\right)\right|^{2} \leq\left|A^{-1 / 4} \mathbf{w}(0)\right|^{2} \exp \left\{-\mu_{1} \eta t_{0} \exp \left(-c_{3} t_{0}\right)+\frac{4 C_{M}^{2}}{3 \mu_{1}} t_{0}\right\} .
$$

We now consider the cases

$$
\left|\mathbf{Q}_{N} \mathbf{A}^{-1 / 4} \mathbf{w}\left(t_{0}\right)\right|>\gamma\left|\mathbf{P}_{N} \mathbf{A}^{-1 / 4} \mathbf{w}\left(t_{0}\right)\right|
$$

and

$$
\left|\mathbf{Q}_{N} \mathbf{A}^{-1 / 4} \mathbf{w}\left(t_{0}\right)\right| \leq \gamma\left|\mathbf{P}_{N} \mathbf{A}^{-1 / 4} \mathbf{w}\left(t_{0}\right)\right|
$$

Then, in view of the statement of Theorem 5.1 (i.e., either (3.4) holds, or (3.5) does), it is necessary only to consider what happens if $(5.20 \mathrm{a})$ applies; in this case

$$
\begin{aligned}
\eta & =\frac{\left|\mathbf{A}^{1 / 4} \mathbf{w}\left(t_{0}\right)\right|^{2}}{\left|\mathbf{A}^{-1 / 4} \mathbf{w}\left(t_{0}\right)\right|^{2}} \\
& =\frac{\left|\mathbf{P}_{N} \mathbf{A}^{1 / 4} \mathbf{w}\left(t_{0}\right)\right|^{2}+\left|\mathbf{Q}_{N} \mathbf{A}^{1 / 4} \mathbf{w}\left(t_{0}\right)\right|^{2}}{\left.\mathbf{P}_{N} \mathbf{A}^{-1 / 4} \mathbf{w}\left(t_{0}\right)\right|^{2}+\left|\mathbf{Q}_{N} \mathbf{A}^{-1 / 4} \mathbf{w}\left(t_{0}\right)\right|^{2}} \\
& \geq \frac{\left|\mathbf{Q}_{N} \mathbf{A}^{1 / 4} \mathbf{w}\left(t_{0}\right)\right|^{2}}{\left(1+\frac{1}{\gamma}\right)\left|\mathbf{Q}_{N} \mathbf{A}^{-1 / 4} \mathbf{w}\left(t_{0}\right)\right|^{2}} \\
& \geq \frac{\gamma}{1+\gamma} \cdot \frac{\lambda_{N+1}^{1 / 2}}{\lambda_{N+1}^{-1 / 2}} \equiv \frac{\gamma}{1+\gamma} \lambda_{N+1},
\end{aligned}
$$

$\lambda_{N+1}$ being the $(N+1)$ st eigenvalue of $\mathbf{A}$. Employing this last lower bound for $\eta$ in (5.19), we are led to the estimate

$$
\begin{aligned}
\left|\mathbf{A}^{-1 / 4} \mathbf{w}\left(t_{0}\right)\right|^{2} & \\
& \leq\left|\mathbf{A}^{-1 / 4} \mathbf{w}(0)\right|^{2} \exp \left\{-\frac{\gamma}{1+\gamma} \mu_{1} \lambda_{N+1} t_{0} \exp \left(-c_{3} t_{0}\right)+\frac{4 C_{M}^{2}}{3 \mu_{1}} t_{0}\right\} \\
& \leq\left|\mathbf{A}^{-1 / 4} \mathbf{w}(0)\right|^{2} \exp \left\{-\frac{\gamma}{1+\gamma} \mu_{1} \lambda_{N+1} t_{0} \exp \left(-c_{3} T\right)+\frac{4 C_{M}^{2}}{3 \mu_{1}} T\right\}
\end{aligned}
$$


as $t_{0}<T$. Replacing $t_{0}$ by $t<T$ in (5.22), we obtain (3.5) with

$$
c_{1}=\exp \left(\frac{4 C_{M}^{2}}{3 \mu_{1}} T\right) \quad \text { and } \quad c_{2}=\frac{\gamma}{\gamma+1} \exp \left(-c_{3} T\right) .
$$

To complete the proof of Theorem 5.1 it remains only to establish (5.1). However, by $(5.13)$,

$$
\begin{gathered}
\frac{d}{d t}\left|\mathbf{A}^{-1 / 4} \mathbf{w}\right|^{2}+4 \mu_{1}\left|\mathbf{A}^{1 / 4} \mathbf{w}\right|^{2} \leq 2 C_{M}\left|\mathbf{A}^{1 / 4} \mathbf{w}\right|\left|\mathbf{A}^{-1 / 4} \mathbf{w}\right| \\
\leq 4 \mu_{1}\left|\mathbf{A}^{1 / 4} \mathbf{w}\right|^{2}+\frac{C_{M I}^{2}}{\mu_{1}}\left|\mathbf{A}^{-1 / 4} \mathbf{w}\right|^{2}
\end{gathered}
$$

so that

$$
\frac{d}{d t}\left|\mathbf{A}^{-1 / 4} \mathbf{w}\right|^{2} \leq \frac{C_{M I}^{2}}{\mu_{1}}\left|\mathbf{A}^{-1 / 4} \mathbf{w}\right|^{2} .
$$

The estimate (5.1) now follows by integrating (5.24) and using the definition of $\mathbf{w}(t)$. The theorem is proved.

6. The fixed point theorem. In this section we will examine both the structure of the space of Lipschitz maps $H_{b, l}(b>0, l>0)$ consisting of those $\phi: \mathbf{P}_{N} D\left(\mathbf{A}^{1 / 4}\right) \rightarrow$ $\mathbf{Q}_{N} D\left(\mathbf{A}^{1 / 4}\right)$ that satisfy (3.8)-(3.10), and also the nature of the mapping $\mathbf{T}$ that associates with each $\phi \in H_{b, l}$ a function $\mathbf{T} \phi$ defined on $\mathbf{P}_{N} D\left(\mathbf{A}^{1 / 4}\right)$. The determination of $\mathbf{T}$ depends on the behavior of solutions of the modified equation (3.7), to which we will turn our attention first, and is detailed in part $\mathrm{V}$ of the procedure outlined in $\S 3$, culminating in the explicit expression (3.21).

As was previously indicated in $\S 3$, it is a straightforward matter to prove the existence and uniqueness of solutions to the modified initial-value problem (3.7), (2.19) for $\mathbf{u}_{0} \in$ $H_{\text {per }}$. The absorbing property of the modified equation may be easily demonstrated by taking the inner product of (3.7) with $\mathbf{A}^{1 / 2} \mathbf{u}$; for $\left|\mathbf{A}^{1 / 4} \mathbf{u}\right| \geq 2 r_{1}$ we obtain

$$
\frac{1}{2} \frac{d}{d t}\left(\mathbf{A}^{1 / 4} \mathbf{u}, \mathbf{A}^{1 / 4} \mathbf{u}\right)+2 \mu_{1}\left(\mathbf{A}^{3 / 4} \mathbf{u}, \mathbf{A}^{3 / 4} \mathbf{u}\right)=0
$$

because $\Theta_{r_{1}}\left(\left|\mathbf{A}^{1 / 4} \mathbf{u}\right|\right)=0$ for $\left|\mathbf{A}^{1 / 4} \mathbf{u}\right| \geq 2 r_{1}$. Inasmuch as $\left(\mathbf{A}^{3 / 4} \mathbf{u}, \mathbf{A}^{3 / 4} \mathbf{u}\right) \geq \lambda_{1}\left(\mathbf{A}^{1 / 4} \mathbf{u}\right.$ $\mathbf{A}^{1 / 4} \mathbf{u}$ ), with $\lambda_{1}>0$ the smallest eigenvalue of $\mathbf{A}$, we have

$$
\frac{1}{2} \frac{d}{d t}\left|\mathbf{A}^{1 / 4} \mathbf{u}\right|^{2}+2 \mu_{1} \lambda_{1}\left|\mathbf{A}^{1 / 4} \mathbf{u}\right|^{2} \leq 0
$$

Thus,

$$
\left|\mathbf{A}^{1 / 4} \mathbf{u}(t)\right|^{2} \leq\left|\mathbf{A}^{1 / 4} \mathbf{u}(0)\right|^{2} e^{-4 \mu_{1} \lambda_{1} t}, \quad t>0 .
$$

Therefore, if $\left|\mathbf{A}^{1 / 4} \mathbf{u}_{0}\right|>r_{2}$, where $r_{2} \geq 2 r_{1}$, the orbit $\mathbf{u}(t)$ will converge exponentially in $D\left(\mathbf{A}^{1 / 4}\right)$ to the ball $B_{r_{2}}$, while if $\left|\mathbf{A}^{1 / 4} \mathbf{u}_{0}\right| \leq r_{2}$, then $\mathbf{u}(t)$ will stay inside the ball $B_{r_{2}}$ for all $t>0$. However, $\Theta_{r_{1}}\left(\left|\mathbf{A}^{1 / 4} \mathbf{u}\right|\right)=1$, for $\left|\mathbf{A}^{1 / 4} \mathbf{u}\right| \leq r_{1}$; so the original equation (2.18) and the modified equation (3.7) are identical in a neighborhood of the global attractor and the dynamics of (2.18) are exactly represented by those of (3.7) after a sufficiently large time. 
We now turn to the proofs of Lemmas 6.1 through 6.6 , full statements of which may be found in $\S 3$.

Proof of Lemma 6.1. The proof follows that of the analogous result in Temam ([15], Lemma $3.1, \S 3.2$ of Chapter VIII) almost without change.

Proof of Lemma 6.2. We want to establish (3.23a,b), where $\mathbf{u}_{i}=\mathbf{p}_{i}+\boldsymbol{\phi}\left(\mathbf{p}_{i}\right), i=1,2$, with $\phi \in H_{b, l}$ and $\mathbf{p}_{1}, \mathbf{p}_{2} \in \mathbf{P}_{N} D\left(\mathbf{A}^{1 / 4}\right)$. However, as a consequence of Lemmas 2.1 and 2.2 of Temam ([15], Chapter VIII), and the Lipschitz property (3.1), it follows that $\exists M_{i}>0, i=1,2$, such that

$$
\begin{gathered}
\left|\mathbf{A}^{-1 / 4} \mathbf{F}\left(\mathbf{u}_{1}\right)\right| \leq M_{1} \\
\left|\mathbf{A}^{-1 / 4}\left(\mathbf{F}\left(\mathbf{u}_{1}\right)-\mathbf{F}\left(\mathbf{u}_{2}\right)\right)\right| \leq M_{2}\left|\mathbf{A}^{1 / 4}\left(\mathbf{u}_{1}-\mathbf{u}_{2}\right)\right|
\end{gathered}
$$

so that $(3.23 \mathrm{a})$ follows. For $(3.23 \mathrm{~b})$ we use $(6.4 \mathrm{~b})$, and the definition of $H_{b, l}$, which implies that

$$
\begin{aligned}
\left|\mathbf{A}^{1 / 4}\left(\mathbf{u}_{1}-\mathbf{u}_{2}\right)\right| & \leq\left|\mathbf{A}^{1 / 4}\left(\mathbf{p}_{1}-\mathbf{p}_{2}\right)\right|+\left|\mathbf{A}^{1 / 4}\left(\phi\left(\mathbf{p}_{1}\right)-\phi\left(\mathbf{p}_{2}\right)\right)\right| \\
& \leq(1+l)\left|\mathbf{A}^{1 / 4}\left(\mathbf{p}_{1}-\mathbf{p}_{2}\right)\right| .
\end{aligned}
$$

Proof of Lemma 6.3. For $\mathbf{p}_{0} \in \mathbf{P}_{N} D\left(\mathbf{A}^{1 / 4}\right)$ we need to establish (3.24). From the definition (3.21) of the mapping $\mathbf{T}$ it is clear that $\mathbf{T} \phi\left(\mathbf{p}_{0}\right) \in \mathbf{Q}_{N} D\left(\mathbf{A}^{1 / 4}\right)$. Also, as a consequence of (3.21) and (3.23a),

$$
\begin{aligned}
& \left|\mathbf{A}^{1 / 4}(\mathbf{T} \phi)\left(\mathbf{p}_{0}\right)\right| \leq \int_{-\infty}^{0}\left|\mathbf{A}^{1 / 4} e^{2 \mu_{1} \mathbf{A} \tau} \mathbf{Q}_{N} \mathbf{F}(\mathbf{p}(\tau)+\phi(\mathbf{p}(\tau)))\right| d \tau \\
& \leq\left(2 \mu_{1}\right)^{1 / 2} \int_{-\infty}^{0}\left\{\left|\left(2 \mu_{1} \mathbf{A} \mathbf{Q}_{N}\right)^{1 / 2} e^{2 \mu_{1} \mathbf{A} \tau}\right|_{\mathcal{L}\left(\mathbf{Q}_{N} H_{\text {per }}\right)}\right. \\
& \left.\quad \times\left|\mathbf{A}^{-1 / 4} \mathbf{F}(\mathbf{p}(\tau)+\boldsymbol{\phi}(\tau))\right|\right\} d \tau \\
& \leq\left(2 \mu_{1}\right)^{1 / 2} M_{1} \int_{-\infty}^{0}\left|\left(2 \mu_{1} \mathbf{A} \mathbf{Q}_{N}\right)^{1 / 2} e^{2 \mu_{1} \mathbf{A} \tau}\right|_{\mathcal{L}\left(\mathbf{Q}_{N} H_{\text {per }}\right)} d \tau
\end{aligned}
$$

However, as a direct consequence of Temam ([15], Lemma 3.2, Chapter VIII) we have the following. Let $\delta \in R^{1}$ and $\tau<0$, and set $k_{2}(\delta)=\delta^{\delta} e^{-\delta}$ and

$$
k_{3}(\delta)= \begin{cases}1, & \text { if } \delta<0, \\ e^{-\delta}+\frac{k_{2}(\delta)}{1-\delta} \cdot \delta^{1-\delta}, & \text { if } 0 \leq \delta<1 .\end{cases}
$$

Then $\left|\left(\mathbf{A} \mathbf{Q}_{N}\right)^{\delta} e^{\tau \mathbf{A} \mathbf{Q}_{N}}\right|$ in $\mathcal{L}\left(\mathbf{Q}_{N} H_{\text {per }}\right)$ is bounded by

$$
\begin{cases}k_{2}(\delta)|\tau|^{-\delta}, & \text { if }-\delta / \lambda_{N+1} \leq \tau<0 \\ \lambda_{N+1}^{\delta} e^{\tau \lambda_{N+1}}, & \text { if } \tau<-\delta /(N+1)\end{cases}
$$

and, moreover, if $\delta<1$,

$$
\int_{-\infty}^{0}\left|\left(\mathbf{A Q}_{N}\right)^{\delta} e^{\tau \mathbf{A} \mathbf{Q}_{N}}\right|_{\mathcal{L}\left(\mathbf{Q}_{N} H_{\text {per }}\right)} d \tau \leq k_{3}(\delta) \lambda_{N+1}^{\delta-1}
$$


Applying (6.7b) with $\delta=\frac{1}{2}$ to the last estimate in (6.6), we have, by virtue of the definitions of $k_{2}(\delta), k_{3}(\delta)$,

$$
\begin{aligned}
\left|\mathbf{A}^{1 / 4}\left(\mathbf{T} \phi\left(\mathbf{p}_{0}\right)\right)\right| & \leq\left(2 \mu_{1}\right)^{-1 / 2} k_{3}\left(\frac{1}{2}\right)\left(2 \mu_{1} \lambda_{N+1}\right)^{-1 / 2} M_{1} \\
& \leq e^{-1 / 2} \mu_{1}^{-1} M_{1} \lambda_{N+1}^{-1 / 2}
\end{aligned}
$$

which completes the proof of (3.24) with $b^{\prime}=e^{-1 / 2} \mu_{1}^{-1} \lambda_{N+1}^{-1 / 2}$; note that $b^{\prime}<b$ for $\lambda_{N+1}$ sufficiently large.

Proof of Lemma 6.4. Given that (3.25) holds, we want to establish the validity of the estimate (3.26), with $l^{\prime}$ given by $(3.27 \mathrm{a}, \mathrm{b}, \mathrm{c})$, for $\phi \in H_{b . l}$ and $\mathbf{p}_{01}, \mathbf{p}_{02} \in \mathbf{p}_{N} D\left(\mathbf{A}^{1 / 4}\right)$. As a direct consequence of this lemma it will follow that $\mathbf{T} \boldsymbol{\phi}$, as defined by (3.21), belongs to the space $H_{b, l^{\prime}}$.

We begin the proof by letting $\phi$ be a fixed but arbitrary element in $H_{b, l}$ and $\mathbf{p}_{1}=\mathbf{p}_{1}(t)$, $\mathbf{p}_{2}=\mathbf{p}_{2}(t)$ solutions of the initial-value problems

$$
\begin{aligned}
& \left\{\begin{array}{l}
\frac{d \mathbf{p}_{1}}{d t}+2 \mu_{1} \mathbf{A} \mathbf{p}_{1}+\mathbf{P}_{N} \mathbf{F}\left(\mathbf{u}_{1}\right)=\mathbf{0} \\
\mathbf{p}_{1}(0)=\mathbf{p}_{01}
\end{array}\right. \\
& \left\{\begin{array}{l}
\frac{d \mathbf{p}_{2}}{d t}+2 \mu_{1} \mathbf{A} \mathbf{p}_{2}+\mathbf{P}_{N} \mathbf{F}\left(\mathbf{u}_{2}\right)=\mathbf{0} \\
\mathbf{p}_{2}(0)=\mathbf{p}_{02}
\end{array}\right.
\end{aligned}
$$

where $\mathbf{u}_{i}=\mathbf{p}_{i}+\phi\left(\mathbf{p}_{i}\right), i=1,2$. Setting $\hat{\mathbf{p}}(t)=\mathbf{p}_{1}(t)-\mathbf{p}_{2}(t)$, we have

$$
\left\{\begin{array}{l}
\frac{d \hat{\mathbf{p}}_{2}}{d t}+2 \mu_{1} \mathbf{A} \hat{\mathbf{p}}+\mathbf{P}_{N}\left(\mathbf{F}\left(\mathbf{u}_{1}\right)-\mathbf{F}\left(\mathbf{u}_{2}\right)\right)=\mathbf{0} \\
\hat{\mathbf{p}}(0)=\mathbf{p}_{01}-\mathbf{p}_{02}
\end{array}\right.
$$

Taking the inner product of the equation for $\hat{\mathbf{p}}(t)$, above, with $\mathbf{A}^{1 / 2} \widehat{\mathbf{p}}$, and applying Lemma 6.2 (specifically, $(3.23 \mathrm{~b})$ ), we obtain

$$
\begin{aligned}
\frac{1}{2} \frac{d}{d t}\left|\mathbf{A}^{1 / 4} \hat{\mathbf{p}}\right|^{2}+2 \mu_{1}\left|\mathbf{A}^{3 / 4} \hat{\mathbf{p}}\right|^{2} & \geq-\left|\mathbf{A}^{-1 / 4}\left(\mathbf{F}\left(\mathbf{u}_{1}\right)-\mathbf{F}\left(\mathbf{u}_{2}\right)\right)\right| \cdot\left|\mathbf{A}^{3 / 4} \hat{\mathbf{p}}\right| \\
& \geq-M_{2}(1+l)\left|\mathbf{A}^{1 / 4} \hat{\mathbf{p}}\right| \cdot\left|\mathbf{A}^{3 / 4} \hat{\mathbf{p}}\right|
\end{aligned}
$$

However,

$$
\left|\mathbf{A}^{3 / 4} \hat{\mathbf{p}}\right|=\left|\mathbf{A}^{1 / 2} \mathbf{A}^{1 / 4} \hat{\mathbf{p}}\right| \leq \lambda_{N}^{1 / 2}\left|\mathbf{A}^{1 / 4} \hat{\mathbf{p}}\right|
$$

so

$$
\left|\mathbf{A}^{1 / 4} \hat{\mathbf{p}}\right| \frac{d}{d t}\left|\mathbf{A}^{1 / 4} \hat{\mathbf{p}}\right|+2 \mu_{1} \lambda_{N}\left|\mathbf{A}^{1 / 4} \hat{p}_{N}\right|^{2} \geq-M_{2}(1+l) \lambda_{N}^{1 / 2}\left|\mathbf{A}^{1 / 4} \hat{\mathbf{p}}\right|^{2}
$$

or

$$
\frac{d}{d t}\left|\mathbf{A}^{1 / 4} \hat{\mathbf{p}}\right|+\left(2 \mu_{1} \lambda_{N}+M_{2}(1+l) \lambda_{N}^{1 / 2}\right)\left|\mathbf{A}^{1 / 4} \hat{\mathbf{p}}\right| \geq 0 .
$$

From (6.14) we easily deduce that, for $\tau \leq 0$,

$$
\left|\mathbf{A}^{1 / 4} \hat{\mathbf{p}}(\tau)\right| \leq\left|\mathbf{A}^{1 / 4} \hat{\mathbf{p}}(0)\right| \exp \left(-\tau\left[2 \mu_{1} \lambda_{N}+M_{2}(1+l) \lambda_{N}^{1 / 2}\right]\right)
$$


Next, using the Lipschitz condition relative to $\mathbf{F}(\mathbf{u})$, which is implied by (3.1), we estimate as follows:

$$
\begin{aligned}
& \left|\mathbf{A}^{1 / 4}\left(\mathbf{T} \boldsymbol{\phi}\left(\mathbf{p}_{01}\right)-\mathbf{T} \boldsymbol{\phi}\left(\mathbf{p}_{02}\right)\right)\right| \\
& \quad \leq \int_{-\infty}^{0}\left|\mathbf{A}^{1 / 4} e^{2 \mu_{1} \mathbf{A} \tau} \mathbf{Q}_{N}\left(\mathbf{F}\left(\mathbf{u}_{1}\right)-\mathbf{F}\left(\mathbf{u}_{2}\right)\right)\right| d \tau \\
& \quad \leq\left(2 \mu_{1}\right)^{-1 / 2} \int_{-\infty}^{0}\left|\left(2 \mu_{1} \mathbf{A} \mathbf{Q}_{N}\right)^{1 / 2} e^{2 \mu_{1} \mathbf{A} \tau}\right| \cdot\left|\mathbf{A}^{-1 / 4}\left(\mathbf{F}\left(\mathbf{u}_{1}\right)-\mathbf{F}\left(\mathbf{u}_{2}\right)\right)\right| d \tau \\
& \quad \leq M_{2}(1+l)\left(2 \mu_{1}\right)^{-1 / 2} \int_{-\infty}^{0}\left|\left(2 \mu_{1} \mathbf{A} \mathbf{Q}_{N}\right)^{1 / 2} e^{2 \mu_{1} \mathbf{A} \tau}\right| \cdot\left|\mathbf{A}^{1 / 4} \hat{\mathbf{p}}(\tau)\right| d \tau \\
& \quad \leq M_{2}(1+l)\left(2 \mu_{1}\right)^{-1 / 2}\left|\mathbf{A}^{1 / 4} \hat{\mathbf{p}}(0)\right| \int_{-\infty}^{0}\left|\left(2 \mu_{1} \mathbf{A} \mathbf{Q}_{N}\right)^{1 / 2} e^{2 \mu_{1} \mathbf{A} \tau}\right| e^{\tau \lambda_{N} \xi_{n}} d t
\end{aligned}
$$

where $\xi_{N}=2 \mu_{1}+M_{2}(1+l) \lambda_{N}^{-1 / 2} ;$ for simplicity we have written $\left|\left(2 \mu_{1} \mathbf{A} \mathbf{Q}_{N}\right)^{1 / 2} e^{2 \mu_{1} \mathbf{A} \tau}\right|$ instead of $\left|\left(2 \mu_{1} \mathbf{A} \mathbf{Q}_{N}\right)^{1 / 2} e^{2 \mu_{1} \mathbf{A} \tau}\right|_{\mathcal{L}\left(\mathbf{Q}_{N} H_{\text {per }}\right)}$, and we have used (6.15), which is valid for $\tau \leq 0$. We now focus our attention on the integral

$$
\int_{-\infty}^{0}\left|\left(2 \mu_{1} \mathbf{A} \phi_{N}\right)^{1 / 2} e^{2 \mu_{1} \mathbf{A} \tau}\right| e^{-\tau \lambda_{N} \xi_{N}} d \tau
$$

in the last estimate of (6.16). By virtue of bounds for $\left|\left(\mathbf{A} \mathbf{Q}_{N}\right)^{\delta} e^{\tau \mathbf{A} \mathbf{Q}_{N}}\right|$ which are given by $(6.7 \mathrm{a})$ we have, first of all, that

$$
\begin{aligned}
& \int_{-\infty}^{-1 /\left(4 \mu_{1} \lambda_{N+1}\right)}\left|\left(2 \mu_{1} \mathbf{A} \mathbf{Q}_{N}\right)^{1 / 2} e^{2 \mu_{1} \mathbf{A} \tau}\right|^{-\tau \lambda_{N} \xi_{N}} d \tau \\
& \quad \leq \int_{-\infty}^{-1 /\left(4 \mu_{1} \lambda_{N+1}\right)}\left(2 \mu_{1} \lambda_{N+1}\right)^{1 / 2} e^{2 \mu_{1} \tau \lambda_{N+1}} e^{-\tau \lambda_{N} \xi_{N}} d \tau \\
& \quad \leq \int_{-\infty}^{-1 /\left(4 \mu_{1} \lambda_{N+1}\right)}\left(2 \mu_{1} \lambda_{N+1}\right)^{1 / 2} e^{-\tau \sigma_{N}} d \tau \\
& \quad \leq\left(2 \mu_{1} \lambda_{N+1}\right)^{1 / 2} \frac{1}{\sigma_{N}} \exp \left[-\frac{\sigma_{N}}{4 \mu_{1} \lambda_{N+1}}\right]
\end{aligned}
$$

where $\sigma_{N}$ is given by $(3.25)$, which is, in fact, equivalent to

$$
\sigma_{N}=\lambda_{N+1}\left(2 \mu_{1}-r_{N} \xi_{N}\right), \quad r_{N}=\lambda_{N} / \lambda_{N+1} .
$$

Therefore,

$$
\begin{aligned}
& \int_{-\infty}^{-1 /\left(4 \mu_{1} \lambda_{N+1}\right)}\left|\left(2 \mu_{1} \mathbf{A} \mathbf{Q}_{N}\right)^{1 / 2} e^{2 \mu_{1} \mathbf{A} \tau}\right| e^{-\tau \lambda_{N} \xi_{N}} d \tau \\
& \quad \leq\left(2 \mu_{1}\right)^{1 / 2} \lambda_{N+1}^{-1 / 2} e^{-1 / 2}\left(2 \mu_{1}-r_{N} \xi_{N}\right)^{-1} \exp \left(\frac{r_{N} \xi_{N}}{4 \mu_{1}}\right)
\end{aligned}
$$


In a like fashion, we have, by again using the bounds for $\left|\left(\mathbf{A} \mathbf{Q}_{N}\right)^{\delta} e^{\tau \mathbf{A} \mathbf{Q}_{N}}\right|$ inherent in (6.7a), the series of estimates

$$
\begin{aligned}
& \int_{-1 /\left(4 \mu_{1} \lambda_{N+1}\right)}^{0}\left|\left(2 \mu_{1} \mathbf{A} \mathbf{Q}_{N}\right)^{1 / 2} e^{2 \mu_{1} \mathbf{A} \tau}\right| e^{-\tau \lambda_{N} \xi_{N}} d \tau \\
& \quad \leq \int_{-1 /\left(4 \mu_{1} \lambda_{N+1}\right)}^{0} k_{2}\left(\frac{1}{2}\right)|\tau|^{-1 / 2} e^{-\tau \lambda_{N} \xi_{N}} d \tau \\
& \quad \leq(2 e)^{-1 / 2} \exp \left(\frac{\lambda_{N} \xi_{N}}{4 \mu_{1} \lambda_{N+1}}\right) \int_{-1 /\left(4 \mu_{1} \lambda_{N+1}\right)}^{0}|\tau|^{-1 / 2} d \tau \\
& \quad=\left(2 \mu_{1}\right)^{-1 / 2} e^{-1 / 2} \lambda_{N+1}^{-1 / 2} \exp \left(\frac{r_{N} \xi_{N}}{4 \mu_{1}}\right) .
\end{aligned}
$$

Combining (6.19) with the last estimate in (6.20), we are led to the bound

$$
\begin{aligned}
& \int_{-\infty}^{0}\left|\left(2 \mu_{1} \mathbf{A} \mathbf{Q}_{N}\right)^{1 / 2} e^{2 \mu_{1} \mathbf{A} \tau}\right| e^{-\tau \lambda_{N} \xi_{N}} d \tau \\
& \quad \leq\left[\left(2 \mu_{1}\right)^{1 / 2}\left(2 \mu_{1}-r_{N} \xi_{N}\right)^{-1}+\left(2 \mu_{1}\right)^{-1 / 2}\right] \lambda_{N+1}^{-1 / 2} e^{-1 / 2} \exp \left(\frac{r_{N} \xi_{N}}{4 \mu_{1}}\right)
\end{aligned}
$$

which, in conjunction with the last estimate in (6.16), i.e.,

$$
\begin{aligned}
& \mid \mathbf{A}^{1 / 4}\left(\mathbf{T} \boldsymbol{\phi}\left(\mathbf{p}_{01}\right)-\mathbf{T} \boldsymbol{\phi}\left(\mathbf{p}_{02}\right) \mid\right. \\
& \quad \leq M_{2}(1+l)\left(2 \mu_{1}\right)^{-1 / 2}\left|\mathbf{A}^{1 / 4} \hat{\mathbf{p}}(0)\right| \int_{-\infty}^{0}\left|\left(2 \mu_{1} \mathbf{A Q}_{N}\right)^{1 / 2} e^{2 \mu_{1} \mathbf{A} \tau}\right| e^{-\tau \lambda_{N} \xi_{N}} d \tau
\end{aligned}
$$

serves to establish (3.26) with $l^{\prime}$ given in $(3.27 \mathrm{a}, \mathrm{b}, \mathrm{c})$.

Proof of Lemma 6.5. We now want to establish that with $\sigma_{N}$ again given by (3.25), and assumed (for now) to be positive, the estimate (3.28) holds for $\phi_{1}, \phi_{2} \in H_{b . l}$ and $\mathbf{p}_{0} \in \mathbf{P}_{N} D\left(\mathbf{A}^{1 / 4}\right)$, provided $L$, in $(3.28)$, is given by

$$
L=\frac{M_{2}}{2 \mu_{1}}\left(2 e^{-1 / 2} \lambda_{N+1}^{-1 / 2}-\lambda_{N}^{-1 / 2} l^{\prime}\right) .
$$

We begin by setting

$$
\mathbf{p}_{i}=\mathbf{p}\left(t, \phi_{i}, \mathbf{p}_{0}\right), \quad \mathbf{u}_{i}=\mathbf{p}_{i}+\phi_{i}\left(\mathbf{p}_{i}\right)
$$

for $i=1,2$, and $\hat{\mathbf{p}}=\mathbf{p}_{1}-\mathbf{p}_{2}$. For $\hat{\mathbf{p}}(t)$ the initial-value problem (6.11) is again applicable, and, thus, so is the first estimate in (6.12). However, if we once again make use of Lemma 6.2 , i.e., of $(6.4 \mathrm{~b})$ as well as $(6.5)$, we have

$$
\begin{aligned}
\left|\mathbf{A}^{-1 / 4}\left(\mathbf{F}\left(\mathbf{u}_{1}\right)-\mathbf{F}\left(\mathbf{u}_{2}\right)\right)\right| \leq M_{2}\left|\mathbf{A}^{1 / 4} \mathbf{u}_{1}-\mathbf{A}^{1 / 4} \mathbf{u}_{2}\right| \\
\leq M_{2}\left(\left|\mathbf{A}^{1 / 4}\left(\mathbf{p}_{1}-\mathbf{p}_{2}\right)\right|+\left|\mathbf{A}^{1 / 4} \boldsymbol{\phi}_{1}\left(\mathbf{p}_{1}\right)-\mathbf{A}^{1 / 4} \boldsymbol{\phi}_{2}\left(\mathbf{p}_{2}\right)\right|\right) \\
\leq M_{2}\left(\left|\mathbf{A}^{1 / 4}\left(\mathbf{p}_{1}-\mathbf{p}_{2}\right)\right|+\left|\mathbf{A}^{1 / 4} \boldsymbol{\phi}_{1}\left(\mathbf{p}_{1}\right)-\mathbf{A}^{1 / 4} \boldsymbol{\phi}_{1}\left(\mathbf{p}_{1}\right)\right|\right. \\
\left.\quad+\left|\mathbf{A}^{1 / 4} \boldsymbol{\phi}_{1}\left(\mathbf{p}_{2}\right)-\mathbf{A}^{1 / 4} \boldsymbol{\phi}_{2}\left(\mathbf{p}_{2}\right)\right|\right) \\
\leq M_{2}\left[(1+l)\left|\mathbf{A}^{1 / 4}\left(\mathbf{p}_{1}-\mathbf{p}_{2}\right)\right|+\left\|\phi_{1}-\boldsymbol{\phi}_{2}\right\|\right] \\
=M_{2}\left[(1+l)\left|\mathbf{A}^{1 / 4} \hat{\mathbf{p}}\right|+\left\|\boldsymbol{\phi}_{1}-\phi_{2}\right\|\right],
\end{aligned}
$$

where $\left\|\phi_{1}-\phi_{2}\right\|$ is given by $(3.11)$. 
Using, once more, the elementary estimate

$$
\left|\mathbf{A}^{3 / 4} \hat{\mathbf{p}}\right|=\left|\mathbf{A}^{1 / 2} \mathbf{A}^{1 / 4} \hat{\mathbf{p}}\right| \leq \lambda_{N}^{1 / 2}\left|\mathbf{A}^{1 / 4} \hat{\mathbf{p}}\right|,
$$

we can now combine the first estimate in (6.12) and the last estimate in (6.23) to produce the differential inequality

$$
\begin{aligned}
& \frac{1}{2} \frac{d}{d t}\left|\mathbf{A}^{1 / 4} \hat{\mathbf{p}}\right|^{2}+2 \mu_{1} \lambda_{N}\left|\mathbf{A}^{1 / 4} \hat{\mathbf{p}}\right|^{2} \\
& \quad \geq-M_{2}(1+l) \lambda_{N}^{1 / 2}\left|\mathbf{A}^{1 / 4} \hat{\mathbf{p}}\right|^{2}-M_{2} \lambda_{N}^{1 / 2}\left\|\phi_{1}-\phi_{2}\right\|\left|\mathbf{A}^{1 / 4} \hat{\mathbf{p}}\right|
\end{aligned}
$$

From (6.24) we easily obtain

$$
\frac{d}{d t}\left|\mathbf{A}^{1 / 4} \hat{\mathbf{p}}\right|+\left(2 \mu_{1} \lambda_{N}+M_{2}(1+l) \lambda_{N}^{1 / 2}\right)\left|\mathbf{A}^{1 / 4} \hat{\mathbf{p}}\right| \geq-M_{2} \lambda_{N}^{1 / 2}\left\|\phi_{1}-\phi_{2}\right\|
$$

However, $\hat{\mathbf{p}}(0)=0$; so integration of $(6.25)$ from zero to $\tau<0$ yields the estimate

$$
\left|\mathbf{A}^{1 / 4} \hat{\mathbf{p}}(\tau)\right| \leq M_{2} \lambda_{N}^{1 / 2}\left(\xi_{N} \lambda_{N}\right)^{-1}\left(\exp \left(-\xi_{N} \lambda_{N} \tau\right)-1\right)\left\|\phi_{1}-\phi_{2}\right\|
$$

where $\xi_{N}=2 \mu_{1}+M_{2}(1+l) \lambda_{N}^{-1 / 2}$. From (6.23) and (6.25) we now deduce the following sequence of estimates (using, once more, the explicit representation of $\mathbf{T}$ given by (3.21)):

$$
\begin{aligned}
\mid \mathbf{A}^{1 / 4} & \left(\mathbf{T} \phi_{1}\left(\mathbf{p}_{0}\right)-\mathbf{T} \phi_{2}\left(\mathbf{p}_{0}\right)\right) \mid \\
\leq & \int_{-\infty}^{0}\left|\mathbf{A}^{1 / 4} e^{2 \mu_{1} \mathbf{A} \tau} \mathbf{Q}_{N}\left(\mathbf{F}\left(\mathbf{u}_{1}\right)-\mathbf{F}\left(\mathbf{u}_{2}\right)\right)\right| d \tau \\
\leq & \left(2 \mu_{1}\right)^{-1 / 2} \int_{-\infty}^{0}\left|\left(2 \mu_{1} \mathbf{A} \mathbf{Q}_{N}\right)^{1 / 2} e^{2 \mu_{1} \mathbf{A} \tau}\right| \cdot\left|\mathbf{A}^{-1 / 4}\left(\mathbf{F}\left(\mathbf{u}_{1}\right)-\mathbf{F}\left(\mathbf{u}_{2}\right)\right)\right| d \tau \\
\leq & \left(2 \mu_{1}\right)^{-1 / 2} M_{2} \int_{-\infty}^{0}\left\{\left|\left(2 \mu_{1} \mathbf{A} \mathbf{Q}_{N}\right)^{1 / 2} e^{2 \mu_{1} \mathbf{A} \tau}\right|\right. \\
\leq & \left(2 \mu_{1}\right)^{-1 / 2} M_{2}\left\|\phi_{1}-\phi_{2}\right\| \\
& \left.\times \int_{-\infty}^{0}\left|\left(2 \mu_{1} \mathbf{A} \mathbf{Q}_{N}\right)^{1 / 2} e^{2 \mu_{1} \mathbf{A} \tau}\right|\left[1+(1+l)\left|\mathbf{A}^{1 / 4} \hat{\mathbf{p}}(t)\right|+\left\|\phi_{1}-\phi_{2}\right\|\right]\right\} d \tau \\
\leq & \left(2 \mu_{1}\right)^{-1 / 2} M_{2}\left\|\phi_{1}-\phi_{2}\right\| \\
& \times\left[\int_{-\infty}^{0}\left|\left(2 \mu_{1} \mathbf{A} \mathbf{Q}_{N}\right)^{1 / 2} e^{2 \mu_{1} \mathbf{A} \tau}\right| d \tau\right. \\
& \left.\quad+\int_{-\infty}^{0}\left|\left(2 \mu_{1} \mathbf{A} \mathbf{Q}_{N}\right)^{1 / 2} e^{2 \mu_{1} \mathbf{A} \tau}\right|(1+l) \frac{M_{2}}{2 \mu_{1}} \lambda_{N}^{-1 / 2} e^{-\tau \lambda_{N} \xi_{N}} d \tau\right] d \tau
\end{aligned}
$$

Applying (6.21), and the bounds for $\left|\left(\mathbf{A} \mathbf{Q}_{N}\right)^{\delta} e^{\tau \mathbf{A} \mathbf{Q}_{N}}\right|$ expressed by (6.7a), to the last estimate above, we deduce that

$$
\begin{aligned}
& \left|\mathbf{A}^{1 / 4}\left(\mathbf{T} \phi_{1}\left(\mathbf{p}_{0}\right)-\mathbf{T} \boldsymbol{\phi}_{2}\left(\mathbf{p}_{0}\right)\right)\right| \\
& \leq\left(2 \mu_{1}\right)^{-1 / 2} M_{2}\left\|\phi_{1}-\phi_{2}\right\| \\
& \quad \times\left[2 e^{-1 / 2}\left(2 \mu_{1}\right)^{-1 / 2} \lambda_{N+1}^{-1 / 2}+\left(\left(2 \mu_{1}\right)^{1 / 2}\left(2 \mu_{1}-\lambda_{N} \xi_{N}\right)^{-1}\right.\right. \\
& \left.\left.\quad+\left(2 \mu_{1}^{-1 / 2}\right)\right) e^{-1 / 2} \lambda_{N+1}^{-1 / 2} e^{r_{N} \xi_{N} /\left(4 \mu_{1}\right)}\right]
\end{aligned}
$$


or

$$
\begin{aligned}
\mid \mathbf{A}^{1 / 4} & \left(\mathbf{T} \boldsymbol{\phi}_{1}\left(\mathbf{p}_{0}\right)-\mathbf{T} \boldsymbol{\phi}_{2}\left(\mathbf{p}_{0}\right)\right) \mid \\
& \leq \frac{M_{2}}{2 \mu_{1}}\left(2 e^{-1 / 2} \lambda_{N+1}^{-1 / 2}+\lambda_{N} l^{\prime}\right)\left\|\phi_{1}-\phi_{2}\right\| \\
& =L\left\|\phi_{1}-\phi_{2}\right\|
\end{aligned}
$$

where

$$
L=\frac{M_{2}}{2 \mu_{1}}\left(2 e^{-1 / 2} \lambda_{N+1}^{-1 / 2}+\lambda_{N}^{-1 / 2} l^{\prime}\right)
$$

and $l^{\prime}$ is given by $(3.27 \mathrm{a})$.

We are now in a position to prove Theorem 6.1 , i.e., that $\mathbf{T}: H_{b, l} \stackrel{\text { into }}{\rightarrow} H_{b, l}$ and is a strict contraction on $H_{b, l}$. From what has been established in Lemmas 6.1-6.5 it follows that we may immediately deduce the validity of Theorem 6.1 once we establish Lemma 6.6 .

Proof of Lemma 6.6. The goal is to show that for $0<l<1$, if

$$
\begin{gathered}
\lambda_{N+1}^{1 / 2}-\lambda_{N}^{1 / 2} \geq k_{1} /\left(2 \mu_{1}\right), \\
\lambda_{N}^{1 / 2} \geq k_{2} /\left(2 \mu_{1}\right),
\end{gathered}
$$

with $k_{1}, k_{2}$ given as per $(3.29)$, then

$$
\sigma_{N}>0, \quad l^{\prime}<l, \quad L<\frac{1}{2} .
$$

The positivity of $\sigma_{N}$ enables us to deduce that the estimate (6.28) is valid, while $l^{\prime}<l$ coupled with (3.26) yields the fact that $\mathbf{T} \boldsymbol{\phi} \in H_{b . l}$ if $\boldsymbol{\phi} \in H_{b, l}$; finally, $L<\frac{1}{2}$ and (6.28) imply that $\mathbf{T}$ is, in fact, a strict contraction on $H_{b, l}$. We begin with the sign of $\sigma_{N}$, and note that the inequality

$$
\sigma_{N}=2 \mu_{1}\left(\lambda_{N+1}-\lambda_{N}\right)-M_{2}(1+l) \lambda_{N}^{1 / 2}>0
$$

is equivalent to the statement that

$$
2 \mu_{1}-r_{N} \xi_{N}>0
$$

where $r_{N}$ and $\xi_{N}$ are given, respectively, by $(3.27 \mathrm{~b}, \mathrm{c})$. If $(6.31)$ holds, however, then

$$
\begin{aligned}
l^{\prime} & =M_{2}(1+l) \lambda_{N+1}^{-1 / 2}\left[\left(2 \mu_{1}\right)^{-1}+\left(2 \mu_{1}-r_{N} \xi_{N}\right)^{-1}\right] e^{-1 / 2} e^{r_{n} \xi_{N} /\left(4 \mu_{1}\right)} \\
& \leq M_{2}(1+l) \lambda_{N+1}^{-1 / 2}\left[\left(2 \mu_{1}\right)^{-1}+\left(2 \mu_{1}-r_{N} \xi_{N}\right)^{-1}\right],
\end{aligned}
$$

in which case, to obtain $l^{\prime}<l$, it suffices to show that

$$
\left(2 \mu_{1}\right)^{-1} M_{2}(1+l) \lambda_{N+1}^{-1 / 2}<l / 2
$$

and

$$
M_{2}(1+l) \lambda_{N+1}^{-1 / 2} \leq \frac{1}{2} l\left(2 \mu_{1}-r_{N} \xi_{N}\right)
$$


Now, (6.33a) can be written in the form

$$
\left(2 \mu_{1}\right)^{-1} k_{1} \leq \lambda_{N+1}^{1 / 2}, \quad k_{1}=2 M_{2}(1+l) l^{-1}
$$

and if $(6.34)$ holds then $(6.33 \mathrm{~b})$ can be written as

$$
k_{1} \lambda_{N+1}^{-1 / 2} \leq 2 \mu_{1}-r_{N} \xi_{N}
$$

or, equivalently, as

$$
k_{1} \lambda_{N+1}^{-1 / 2}-2 \mu_{1}+2 \mu_{1} r_{N}+M_{2}(1+l) \lambda_{N+1}^{-1 / 2} r_{N}^{1 / 2} \leq 0
$$

Assuming that (6.29a) holds or, equivalently, that

$$
2 \mu_{1} r_{N}^{1 / 2}+k_{1} \lambda_{N+1}^{-1 / 2} \leq 2 \mu_{1}
$$

we find that (6.35) holds, i.e.,

$$
\begin{aligned}
& k_{1} \lambda_{N+1}^{-1 / 2}-2 \mu_{1}+2 \mu_{1} r_{N}+M_{2}(1+l) \lambda_{N+1}^{-1 / 2} r_{N}^{1 / 2} \\
& \quad \leq k_{1} \lambda_{N+1}^{-1 / 2}-2 \mu_{1}+2 \mu_{1} r_{N}+k_{1} \lambda_{N+1}^{-1 / 2} r_{N}^{1 / 2} \\
& \quad \leq k_{1} \lambda_{N+1}^{-1 / 2}-2 \mu_{1}+2 \mu_{1} r_{N}^{1 / 2} \leq 0 .
\end{aligned}
$$

However, it is easily seen that both (6.31) and (6.34) are direct consequences of the spectral gap condition (6.29a). Thus if (6.29a) holds, then both $\sigma_{N}>0$ and $l^{\prime}<l$. The precise sequence of steps delineated above may be ordered as follows:

(i) $(6.29 \mathrm{a}) \Rightarrow(6.31) \Rightarrow l^{\prime}<l$ if $(6.33 \mathrm{a})$ and $(6.33 \mathrm{~b})$ hold.

(ii) $(6.29 \mathrm{a}) \Rightarrow(6.34) \Leftrightarrow(6.33 \mathrm{a})$.

(iii) $(6.29 \mathrm{a}) \Leftrightarrow(6.36) \Rightarrow(6.35) \Leftrightarrow(6.33 \mathrm{~b})$.

Finally, in order to show that $L<1$, so that $\mathbf{T}$ is, in fact, by virtue of the estimate (6.28), a contraction map on the complete metric space $H_{b, l}$, it suffices to demonstrate that

$$
L=\frac{M_{2}}{2 \mu_{1}}\left(2 e^{-1 / 2} \lambda_{N+q}^{-1 / 2}+\lambda_{N}^{-1 / 2} l^{\prime}\right)<\frac{1}{2} .
$$

Since $l^{\prime}<l$, however, and $\lambda_{N+1}^{1 / 2} \geq \lambda_{N}^{1 / 2}$,

$$
L<\frac{M_{2}}{2 \mu_{1}}\left(2 e^{-1 / 2}+l\right) \lambda_{N}^{-1 / 2}<\frac{1}{2}
$$

by virtue of the hypothesis $(6.29 \mathrm{~b})$ and the explicit form of $k_{2}$ as given in (3.29). This completes the proof of Lemma 6.6 and, as a direct consequence, the proof of Theorem 6.1 as stated in $\S 3$. 
7. Existence of the inertial manifold. In this section we will complete the proof of the existence of an inertial manifold for the bipolar viscous equations by demonstrating the validity of Theorem 7.1, as stated in $\S 3$. As a consequence of Theorem 6.1 , we know that the mapping $\mathbf{T}$ is, in fact, a (contraction) map of the complete metric space $H_{b, l}$ into itself provided $\left(6.29 \mathrm{a}\right.$,b) hold with $k_{1}, k_{2}$ given by $(3.29)$; thus $\mathbf{T}$ has a fixed point $\phi^{*} \in H_{b, l}$, and we want to show that $\mathcal{M} \equiv$ graph $\phi^{*}$ is the required inertial manifold.

From the definition of $H_{b, l}$ it should be clear that $\mathcal{M}$ is a finite-dimensional Lipschitz manifold, and it is an easy exercise to show directly that $\mathcal{M}$ is invariant under the action of the solution operator $S_{\mu_{1}}(t)$, i.e., $S_{\mu_{1}}(t) \mathcal{M} \subset \mathcal{M}$. Therefore, to complete the proof that $\mathcal{M}$ is an inertial manifold for the bipolar viscous equations it suffices to show that $\mathcal{M}$ attracts exponentially all orbits of the modified initial-value problem (3.7), (2.19), i.e., that, for $\mathbf{u}_{0} \in D\left(\mathbf{a}^{1 / 4}\right), \exists t_{0}>0$ such that for $t \geq t_{0}$ the estimate (3.30) applies; this will be accomplished by making use of the squeezing property for the orbits of (3.7), (2.19).

Proof of Theorem 7.1. We begin by noting that it is a straightforward matter to establish a squeezing property for orbits of the modified problem (3.7), (2.91) which is entirely analogous to the one proven in Theorem 5.1 for the initial-value problem (2.18), (2.19); more specifically, for solutions $\mathbf{u}(t), \mathbf{v}(t)$ of $(3.7),(2.19)$ satisfying (3.3) for some $M>0$, if we are given $\gamma>0$, then, for any $t \in[0, T]$ and every $N, \exists \bar{c}_{i}, i=1,2$, such that either (3.4) holds or (3.5) does with $\bar{c}_{i}$ replacing $c_{i}, i=1,2$. For the orbits of (3.7), (2.19) satisfying (3.3), for some $M>0$, (5.1) will also hold, for $C_{M}$ replaced by some $\bar{C}_{M}>0$, and $t \in[0, T]$; thus, setting

$$
t_{0}=\min \left(\frac{\mu_{1} \ln 2}{\bar{C}_{M}^{2}}, \frac{T}{2}\right)
$$

we obtain, from this modified version of (5.1), the estimate

$$
\left|\mathbf{A}^{-1 / 4}(\mathbf{u}(t)-\mathbf{v}(t))\right| \leq 2\left|\mathbf{A}^{-1 / 4}(\mathbf{u}(0)-\mathbf{v}(0))\right|, \quad t<2 t_{0} .
$$

If we set $\gamma=\frac{1}{8}$ and choose $N>N_{0}$, where $N_{0}$ satisfies

$$
\lambda_{N_{0}+1} \geq\left(\bar{c}_{1} \mu_{1} t_{0}\right)^{-1} \ln \left(2 \bar{c}_{2}\right)
$$

then from the modified forms of $(3.4),(3.5)$ we will have either

$$
\left|\mathbf{Q}_{N} \mathbf{A}^{-1 / 4}(\mathbf{u}(t)-\mathbf{v}(t))\right| \leq \frac{1}{8}\left|\mathbf{P}_{N} \mathbf{A}^{-1 / 4}(\mathbf{u}(t)-\mathbf{v}(t))\right|
$$

or

$$
\left|\mathbf{A}^{-1 / 4}(\mathbf{u}(t)-\mathbf{v}(t))\right| \leq \frac{1}{2}\left|\mathbf{A}^{-1 / 4}(\mathbf{u}(0)-\mathbf{v}(0))\right|
$$

where $\mathbf{u}_{0}, \mathbf{v}_{0} \in D\left(\mathbf{A}^{1 / 4}\right),\left|\mathbf{A}^{1 / 4} \mathbf{u}(0)\right| \leq M,\left|\mathbf{A}^{1 / 4} \mathbf{v}(0)\right| \leq M$, and $t_{0} \leq t \leq 2 t_{0}$.

We now denote the distance between any point $\mathbf{w}$ in the absorbing ball $B_{r_{2}}$ in $D\left(\mathbf{A}^{1 / 4}\right)$ and the manifold $\mathcal{M}$ by

$$
\operatorname{dist}(\mathbf{w}, \mathcal{M})=\inf _{\mathbf{v} \in \mathcal{M}}\left\{\left|\mathbf{A}^{-1 / 4}(\mathbf{w}-\mathbf{v})\right|\right\}
$$


To show that $\mathcal{M}$ attracts all orbits of the modified initial-value problem (3.7), (2.19) exponentially, it suffices to prove that $\mathcal{M}$ attracts, exponentially, all orbits contained in the absorbing ball $B_{r_{2}}$, i.e., all orbits $\mathbf{u}(t)$ such that $\left|\mathbf{A}^{1 / 4} \mathbf{u}(t)\right| \leq r_{2}, t \in[0, \infty)$. So, let $\mathbf{v}(0)=\mathbf{v}_{0} \in \mathcal{M}, \mathbf{v}_{0}=\mathbf{P}_{N} \mathbf{v}_{0}+\phi\left(\mathbf{P}_{N} \mathbf{v}_{0}\right)$, be such that

$$
\left.\operatorname{dist}(\mathbf{u}(0), \mathcal{M})=\mid \mathbf{A}^{-1 / 4} \mathbf{u}(0)-\mathbf{v}(0)\right) \mid
$$

Obviously,

$$
\left|\mathbf{P}_{N} \mathbf{A}^{1 / 4} \mathbf{v}(0)\right| \leq r_{2}
$$

so that, with $b>0$ as in the definition of $H_{b, l}$,

$$
\left|\mathbf{A}^{1 / 4} \mathbf{v}(0)\right| \leq r_{2}+b
$$

in which case, for $t \geq 0$,

$$
\left|\mathbf{A}^{1 / 4} \mathbf{v}(t)\right|=\left|\mathbf{A}^{1 / 4} S_{\mu_{1}}(t) \mathbf{v}(0)\right| \leq r_{2}+b, \quad \forall t \geq 0
$$

Choosing $M>0$ (in the statement of the squeezing property for the orbits of (3.7), (2.19)) to be $M=r_{2}+b$, we apply the estimates recorded in (7.4), (7.5) to $S_{\mu_{1}}\left(t_{1}\right) \mathbf{u}_{0}$ and $S_{\mu_{1}}\left(t_{1}\right) \mathbf{v}_{0}$, with $t_{0} \leq t_{1} \leq 2 t_{0}$ : if (7.5) applies, then

$$
\begin{aligned}
\operatorname{dist}\left(S_{\mu_{1}}\left(t_{1}\right) \mathbf{u}_{0}, \mathcal{M}\right) & \leq\left|\mathbf{A}^{-1 / 4}\left(S_{\mu_{1}}\left(t_{1}\right) \mathbf{u}_{0}-S_{\mu_{1}}\left(t_{1}\right) \mathbf{v}_{0}\right)\right| \\
& \leq \frac{1}{2}\left|\mathbf{A}^{-1 / 4}\left(\mathbf{u}_{0}-\mathbf{v}_{0}\right)\right|=\frac{1}{2} \operatorname{dist}\left(\mathbf{u}_{0}, \mathcal{M}\right)
\end{aligned}
$$

On the other hand, if (7.4) holds, then we have the following sequence of estimates:

$$
\begin{aligned}
\operatorname{dis} & \left(S_{\mu_{1}}\left(t_{1}\right) \mathbf{u}_{0}, \mathcal{M}\right) \\
& \leq\left|\mathbf{A}^{-1 / 4}\left(S_{\mu_{1}}\left(t_{1}\right) \mathbf{u}_{0}-\left(\mathbf{P}_{N} S_{\mu_{1}}\left(t_{1}\right) \mathbf{v}_{0}+\phi\left(\mathbf{P}_{N} S_{\mu_{1}}\left(t_{1}\right) \mathbf{v}_{0}\right)\right)\right)\right| \\
& \leq\left|\mathbf{A}^{-1 / 4}\left(\mathbf{Q}_{N} S_{\mu_{1}}\left(t_{1}\right) \mathbf{u}_{0}-\phi\left(\mathbf{P}_{N} S_{\mu_{1}}\left(t_{1}\right) \mathbf{v}_{0}\right)\right)\right| \\
& \leq\left|\mathbf{A}^{-1 / 4}\left(\mathbf{Q}_{N} S_{\mu_{1}}\left(t_{1}\right) \mathbf{u}_{0}-\mathbf{Q}_{N} S_{\mu_{1}}\left(t_{1}\right) \mathbf{v}_{0}\right)\right| \\
& \leq\left|\mathbf{A}^{-1 / 4}\left(\boldsymbol{\phi}\left(\mathbf{P}_{N} S_{\mu_{1}}\left(t_{1}\right) \mathbf{u}_{0}-\boldsymbol{\phi}\left(\mathbf{P}_{N} S_{\mu_{1}}\left(t_{1}\right) \mathbf{v}_{0}\right)\right)\right)\right| \\
& \leq\left(l+\frac{1}{8}\right)\left|\mathbf{A}^{-1 / 4}\left(\mathbf{P}_{N} S_{\mu_{1}}\left(t_{1}\right) \mathbf{u}_{0}-\mathbf{P}_{N} S_{\mu_{1}}\left(t_{1}\right) \mathbf{v}_{0}\right)\right|
\end{aligned}
$$

Taking $l=\frac{1}{8}$ in the last estimate, we find that

$$
\begin{aligned}
\operatorname{dist}\left(S_{\mu_{1}}\left(t_{1}\right) \mathbf{u}_{0}, \mathcal{M}\right) & \leq \frac{1}{4}\left|\mathbf{A}^{-1 / 4}\left(S_{\mu_{1}}\left(t_{1}\right) \mathbf{u}_{0}-S_{\mu_{1}}\left(t_{1}\right) \mathbf{v}_{0}\right)\right| \\
& \leq \frac{1}{4} \cdot 2\left|\mathbf{A}^{-1 / 4}\left(\mathbf{u}_{0}-\mathbf{v}_{0}\right)\right| \leq \frac{1}{2} \operatorname{dist}\left(\mathbf{u}_{0}, \mathcal{M}\right)
\end{aligned}
$$

for $t_{0} \leq t_{1} \leq 2 t_{0}$. Iterating upon the procedure delineated above, we have, therefore, for $t_{0} \leq t_{1} \leq 2 t_{0}$

$$
\operatorname{dist}\left(S_{\mu_{1}}\left(n t_{1}\right) \mathbf{u}_{0}, \mathcal{M}\right) \leq\left(\frac{1}{2}\right)^{n} \operatorname{dist}\left(\mathbf{u}_{0}, \mathcal{M}\right) \rightarrow 0
$$


as $n \rightarrow \infty$. For arbitrary $t \geq t_{0}$ we may write $t=n t_{1}$ for some $t_{1}, t_{0} \leq t_{1} \leq 2 t_{0}$, in which case

$$
\begin{aligned}
\operatorname{dist}\left(S_{\mu_{1}}(t) \mathbf{u}_{0}, \mathcal{M}\right) & \leq\left(\frac{1}{2}\right)^{n} \operatorname{dist}\left(u_{0}, \mathcal{M}\right) \\
& \leq \exp \left(-\frac{t}{t_{1}} \ln 2\right) \operatorname{dist}\left(\mathbf{u}_{0}, \mathcal{M}\right) \\
& \leq \exp \left(-\frac{t}{2 t_{0}} \ln 2\right) \operatorname{dist}\left(\mathbf{u}_{0}, \mathcal{M}\right)
\end{aligned}
$$

thus establishing the required exponential convergence of orbits of the modified initialvalue problem (3.7), (2.19) - and, hence, of the original problem (2.18), (2.19), as well - to the manifold $\mathcal{M}$ that is generated as the graph of the unique fixed point of $\mathbf{T}$. The proof of the existence of an inertial manifold for the space-periodic version of the nonlinear, incompressible, bipolar viscous model is now complete.

Acknowledgment. The work contained in this paper is based, in large measure, on the Ph.D. thesis of W. Hao. The authors wish to express their gratitude to Professors H. Bellout and J. Nečas for a number of useful conversations connected with this research.

Appendix: The spectral gap condition. We will examine, here, the validity of the spectral gap condition (6.29a) with respect to the operator $\mathbf{A}$ as defined by (2.3), (2.4). Thus, let $\Omega=[0, L]^{n}, L>0, n=2,3$, and define $H_{\text {per }}(\Omega)$ and $V_{\text {per }}(\Omega)$ as in (1.16). We consider, in $V_{\text {per }}(\Omega)$, the eigenvalue problem

$$
\left\{\begin{array}{c}
\Delta \Delta \mathbf{u}+\Delta p=\lambda \mathbf{u} \\
\nabla \cdot \mathbf{u}=0
\end{array}\right.
$$

Definition. The number $\lambda$ is an eigenvalue of $\mathbf{A}$ if $\exists \mathbf{u} \in V_{\text {per }}(\Omega), \mathbf{u} \neq \mathbf{0}$, such that

$$
\int_{\Omega} \Delta \mathbf{u} \cdot \Delta \mathbf{v} d \mathbf{x}=\lambda \int_{\Omega} \mathbf{u} \cdot \mathbf{v} d \mathbf{x}, \forall \mathbf{v} \in V_{\text {per }}(\Omega)
$$

Of course, $\mathbf{u}$ is then called an eigenfunction of $\mathbf{A}$ corresponding to the eigenvalue $\lambda$. We begin with the case $n=3$.

LEMMA A1. If $n=3$, then the numbers

$$
\left\{\frac{16 \pi^{4}}{L^{4}}\left(n_{1}^{2}+n_{2}^{2}+n_{3}^{2}\right) \neq 0 \mid n_{i}, i=1,2,3 \text { are nonnegative integers }\right\}
$$

are eigenvalues of $\mathbf{A}$.

Proof. Let $n_{1}, n_{2}, n_{3}$ be three nonnegative integers with $n_{1}^{2}+n_{2}^{2}+n_{3}^{2} \neq 0$. We first show that $\lambda=\left(16 \pi^{4} / L^{4}\right)\left(n_{1}^{2}+n_{2}^{2}+n_{3}^{2}\right)$ is an eigenvalue of $\mathbf{A}$ with corresponding eigenfunction $\mathbf{u}$ of the form

$$
u_{i}=\sum_{j=1}^{8} C_{i j} f_{j}\left(x_{1}, x_{2}, x_{3}\right), \quad i=1,2,3
$$


where

$$
\left\{\begin{array}{l}
f_{1}\left(x_{1}, x_{2}, x_{3}\right)=\cos \frac{2 \pi n_{1}}{L} x_{1} \cos \frac{2 \pi n_{2}}{L} x_{2} \cos \frac{2 \pi n_{3}}{L} x_{3} \\
f_{2}\left(x_{1}, x_{2}, x_{3}\right)=\cos \frac{2 \pi n_{1}}{L} x_{1} \cos \frac{2 \pi n_{2}}{L} x_{2} \sin \frac{2 \pi n_{3}}{L} x_{3} \\
f_{3}\left(x_{1}, x_{2}, x_{3}\right)=\cos \frac{2 \pi n_{1}}{L} x_{1} \sin \frac{2 \pi n_{2}}{L} x_{2} \cos \frac{2 \pi n_{3}}{L} x_{3} \\
f_{4}\left(x_{1}, x_{2}, x_{3}\right)=\cos \frac{2 \pi n_{1}}{L} x_{1} \sin \frac{2 \pi n_{2}}{L} x_{2} \sin \frac{2 \pi n_{3}}{L} x_{3} \\
f_{5}\left(x_{1}, x_{2}, x_{3}\right)=\sin \frac{2 \pi n_{1}}{L} x_{1} \cos \frac{2 \pi n_{2}}{L} x_{2} \cos \frac{2 \pi n_{3}}{L} x_{3} \\
f_{6}\left(x_{1}, x_{2}, x_{3}\right)=\sin \frac{2 \pi n_{1}}{L} x_{1} \cos \frac{2 \pi n_{2}}{L} x_{2} \sin \frac{2 \pi n_{3}}{L} x_{3} \\
f_{7}\left(x_{1}, x_{2}, x_{3}\right)=\sin \frac{2 \pi n_{1}}{L} x_{1} \sin \frac{2 \pi n_{2}}{L} x_{2} \cos \frac{2 \pi n_{3}}{L} x_{3} \\
f_{8}\left(x_{1}, x_{2}, x_{3}\right)=\sin \frac{2 \pi n_{1}}{L} x_{1} \sin \frac{2 \pi n_{2}}{L} x_{2} \sin \frac{2 \pi n_{3}}{L} x_{3}
\end{array}\right.
$$

By direct calculation, (A3) and (A4) lead to

$$
\begin{aligned}
& \frac{\partial u_{i}}{\partial x_{1}}=\frac{2 \pi n_{1}}{L}\left\{-C_{i 1} f_{5}-C_{i 2} f_{6}-C_{i 3} f_{7}-C_{i 4} f_{8}\right. \\
& \left.+C_{i 5} f_{1}+C_{i 6} f_{2}+C_{i 7} f_{3}+C_{i 8} f_{4}\right\}, \\
& \frac{\partial u_{i}}{\partial x_{2}}=\frac{2 \pi n_{2}}{L}\left\{-C_{i 1} f_{3}-C_{i 2} f_{4}+C_{i 3} f_{1}+C_{i 4} f_{2}\right. \\
& \left.-C_{i 5} f_{7}-C_{i 6} f_{8}+C_{i 7} f_{5}+C_{i 8} f_{6}\right\} \text {, } \\
& \frac{\partial u_{i}}{\partial x_{3}}=\frac{2 \pi n_{3}}{L}\left\{-C_{i 1} f_{2}+C_{i 2} f_{1}-C_{i 3} f_{4}-C_{i 4} f_{3}\right. \\
& \left.-C_{i 5} f_{6}+C_{i 6} f_{5}-C_{i 7} f_{8}+C_{i 8} f_{7}\right\}
\end{aligned}
$$

so that for $i, j=1,2,3$ it is easily verified that

$$
\frac{\partial^{2} u_{i}}{\partial x_{j}^{2}}=-\left(\frac{2 \pi n_{j}}{L}\right)^{2} u_{i}
$$

Therefore,

$$
\Delta \Delta u_{i}=\left(\frac{2 \pi}{L}\right)^{4}\left(n_{1}^{2}+n_{2}^{2}+n_{3}^{2}\right)^{2} u_{i}, \quad i=1,2,3
$$


Now, employing (A5)-(A7), we have

$$
\begin{aligned}
\nabla \cdot \mathbf{u} \equiv \sum_{i=1}^{3} \frac{\partial u_{i}}{\partial x_{i}}= & \frac{2 \pi}{L}\left(n_{1} C_{15}+n_{2} C_{23}+n_{3} C_{32}\right) f_{1} \\
& +\frac{2 \pi}{L}\left(n_{1} C_{16}+n_{2} C_{24}-n_{3} C_{31}\right) f_{2} \\
& +\frac{2 \pi}{L}\left(n_{1} C_{17}-n_{2} C_{21}+n_{3} C_{34}\right) f_{3} \\
& +\frac{2 \pi}{L}\left(n_{1} C_{18}-n_{2} C_{22}-n_{3} C_{33}\right) f_{4} \\
& +\frac{2 \pi}{L}\left(-n_{1} C_{11}+n_{2} C_{27}+n_{3} C_{36}\right) f_{5} \\
& +\frac{2 \pi}{L}\left(-n_{1} C_{12}+n_{2} C_{28}-n_{3} C_{35}\right) f_{6} \\
& +\frac{2 \pi}{L}\left(-n_{1} C_{13}-n_{2} C_{25}+n_{3} C_{38}\right) f_{7} \\
& +\frac{2 \pi}{L}\left(-n_{1} C_{14}-n_{2} C_{26}+n_{3} C_{37}\right) f_{8} .
\end{aligned}
$$

For $n_{1}^{2}+n_{2}^{2}+n_{3}^{2} \neq 0$ the condition $\nabla \cdot \mathbf{u}=0$ yields the algebraic system

$$
\left\{\begin{array}{l}
n_{1} C_{15}+n_{2} C_{23}+n_{3} C_{32}=0, \\
n_{1} C_{16}+n_{2} C_{24}-n_{3} C_{31}=0, \\
n_{1} C_{17}-n_{2} C_{21}+n_{3} C_{34}=0, \\
n_{1} C_{18}-n_{2} C_{22}-n_{3} C_{33}=0, \\
-n_{1} C_{11}+n_{2} C_{27}+n_{3} C_{36}=0 \\
-n_{1} C_{12}+n_{2} C_{28}-n_{3} C_{35}=0 \\
-n_{1} C_{13}+n_{2} C_{25}+n_{33} C_{38}=0 \\
-n_{1} C_{14}-n_{2} C_{26}-n_{33} C_{37}=0
\end{array}\right.
$$

Since (A11) is a homogeneous system of eight equations in twenty-four unknowns, it follows that $\left(16 \pi^{4} / L^{4}\right)\left(n_{1}^{2}+n_{2}^{2}+n_{3}^{2}\right)$ is an eigenvalue of $\mathbf{A}$ with sixteen corresponding independent eigenfunctions of the form given by (A3), (A4). Next, if $n_{1}=0$ but $n_{2}^{2}+n_{3}^{2} \neq$ 0 , then by virtue of $\left(A_{3}\right)$ we will have $f_{5}=f_{6}=f_{7}=f_{8}=0$. By virtue of $(\mathrm{A} 9), \nabla \cdot \mathbf{u}=0$ now implies that

$$
\left\{\begin{array}{l}
n_{2} C_{23}+n_{33} C_{32}=0 \\
n_{2} C_{24}-n_{33} C_{31}=0 \\
-n_{2} C_{21}+n_{3} C_{34}=0 \\
-n_{2} C_{22}-n_{3} C_{33}=0
\end{array}\right.
$$

Therefore, as a consequence of (A9) and (A12) it follows that, with $n_{1}=0$ and $n_{2}^{2}+n_{3}^{2} \neq$ $0,\left(16 \pi^{4} / L^{4}\right)\left(n_{1}^{2}+n_{2}^{2}+n_{3}^{2}\right)$ is an eigenvalue of $\mathbf{A}$ with eight corresponding independent eigenfunctions of the form (A3). In an entirely analogous manner we can show that if $n_{2}=0, n_{1}^{2}+n_{3}^{2} \neq 0$, or $n_{3}=0, n_{1}^{2}, n_{2}^{2} \neq 0$, then $\left(16 \pi^{4} / L^{4}\right)\left(n_{1}^{2}+n_{2}^{2}+n_{3}^{2}\right)$ is still an eigenvalue of $\mathbf{A}$ with eight corresponding independent eigenfunctions of the form (A3). Finally, if $n_{1}=n_{2}=0, n_{3} \neq 0$, then $f_{3}=f_{4}=f_{5}=f_{7}=f_{8}=0$, while $\nabla \cdot \mathbf{u}=0$ implies that

$$
n_{3} C_{32}=0,-n_{3} C_{31}=0 \Leftrightarrow C_{32}=C_{31}=0 .
$$


Thus, for $n_{1}=n_{2}=0$ and $n_{3} \neq 0,\left(16 \pi^{4} / L^{4}\right)\left(n_{1}^{2}+n_{2}^{2}+n_{3}^{2}\right)$ is an eigenvalue of $\mathbf{A}$ with four corresponding, independent, eigenfunctions of the form (A3); the analogous result holds for the remaining two cases, i.e., $n_{1}=n_{3}=0, n_{2} \neq 0$, or $n_{2}=n_{3}=0, n_{1} \neq 0$, and the proof of the lemma is complete.

LEMMA A2. The set of numbers

$$
\left\{\frac{16 \pi^{4}}{L^{4}}\left(n_{1}^{2}+n_{2}^{2}+n_{3}^{2}\right) \neq 0 \mid n_{i}, i=1,2,3 \text { are nonnegative integers }\right\}
$$

exhausts all of the eigenvalues of $\mathbf{A}$.

Proof. Suppose that $\lambda$ is an eigenvalue of $\mathbf{A}$ and $\mathbf{u} \neq \mathbf{0}$ is a corresponding eigenfunction (in $V_{\text {per }}(\Omega)$ ). Then $\mathbf{u}$ possesses the Fourier expansion

$$
\mathbf{u}=\sum_{\substack{n_{1}, n_{2}, n_{3} \\ \text { nonnegative integers }}} \mathbf{u}_{\left(n_{1}, n_{2}, n_{3}\right)}
$$

with each $\mathbf{u}_{\left(n_{1}, n_{2}, n_{3}\right)}$ of the form (A3), (A4). If $\mathbf{u} \in V_{\text {per }}(\Omega)$, then

$$
\int_{\Omega} \mathbf{u} d \mathbf{x}=\mathbf{0}
$$

and $\forall \mathbf{n} \neq \mathbf{0}, \mathbf{n}=\left(n_{1}, n_{2}, n_{3}\right)$, we also have

$$
\int_{\Omega} \mathbf{u}_{\left(n_{1}, n_{2}, n_{3}\right)} d \mathbf{x}=\mathbf{0}, \quad \mathbf{n} \neq \mathbf{0} .
$$

Therefore, by virtue of (A14) and (A15),

$$
\int_{\Omega} \mathbf{u}_{(0,0,0)} d \mathbf{x}=\int_{\Omega} \mathbf{u} d \mathbf{x}-\int_{\Omega} \sum_{\mathbf{n} \neq \mathbf{0}} \mathbf{u}_{\left(n_{1}, n_{2}, n_{3}\right)} d \mathbf{x}
$$

so that

$$
\int_{\Omega} \mathbf{u}_{(0,0,0)} d \mathbf{x}=0
$$

Since $\mathbf{u}_{(0,0,0)}$ is a constant vector, it follows from (A18) that $\mathbf{u}_{(0,0,0)}=\mathbf{0}$. Also, since we require that $\nabla \cdot \mathbf{u}=0, \forall \mathbf{n}=\left(n_{1}, n_{2}, n_{3}\right)$, with the $n_{j}$ nonnegative integers, $\nabla \cdot \mathbf{u}_{\left(n_{1}, n_{2}, n_{3}\right)}=$ 0 ; that this last statement is true follows from the fact that

$$
\begin{aligned}
\nabla \cdot \mathbf{u}=\mathbf{0} & \Rightarrow \int_{\Omega}(\nabla \cdot \mathbf{u})^{2} d \mathbf{x}=0 \\
& \Rightarrow \sum_{\mathbf{n}=\left(n_{1}, n_{2}, n_{3}\right)} \int_{\Omega}\left(\nabla \cdot \mathbf{u}_{\left(n_{1}, n_{2}, n_{3}\right)}\right)^{2} d \mathbf{x}=0
\end{aligned}
$$

as well as the fact that, by virtue of (A3) and (A4), for $\mathbf{n} \neq \mathbf{n}^{\prime}$,

$$
\int_{\Omega}\left(\nabla \cdot \mathbf{u}_{\left(n_{1}, n_{2}, n_{3}\right)}\right) \cdot\left(\nabla \cdot \mathbf{u}_{\left(n_{1}^{\prime}, n_{2}^{\prime}, n_{3}^{\prime}\right)}\right) d \mathbf{x}=0 .
$$


Since $\nabla \cdot \mathbf{u}_{\left(n_{1}, n_{2}, n_{3}\right)}=0$, a.e. in $\Omega$, we have $\mathbf{u}_{\left(n_{1}, n_{2}, n_{3}\right)} \in V_{\text {per }}(\Omega), \forall \mathbf{n} \neq \mathbf{0}$. However, $\mathbf{u} \neq \mathbf{0}$ implies that there exists $\left(n_{1}^{\prime}, n_{2}^{\prime}, n_{3}^{\prime}\right) \neq \mathbf{0}$ such that $\mathbf{u}_{\left(n_{1}, n_{2}, n_{3}\right)} \neq \mathbf{0}$. We now claim that $\lambda$ must be of the form

$$
\lambda=\frac{16 \pi^{4}}{L^{4}}\left(n_{1}^{\prime 2}, n_{2}^{\prime 2}, n_{3}^{\prime 2}\right) ;
$$

to see this we note that, by virtue of the definition given above,

$$
\int_{\Omega} \Delta \mathbf{u} \cdot \Delta \mathbf{v} d \mathbf{x}=\lambda \int_{\Omega} \mathbf{u} \cdot \mathbf{v} d \mathbf{x}, \quad \forall \mathbf{v} \in V_{\operatorname{per}(\Omega)}
$$

so, taking $\mathbf{v}=\mathbf{u}_{\left(n_{1}^{\prime}, n_{2}^{\prime}, n_{3}^{\prime}\right)}$, we obtain

$$
\int_{\Omega} \Delta \mathbf{u} \cdot \Delta \mathbf{u}_{\left(n_{1}^{\prime}, n_{2}^{\prime}, n_{3}^{\prime}\right)} d \mathbf{x}=\lambda \int_{\Omega} \mathbf{u} \cdot \mathbf{u}_{\left(n_{1}^{\prime}, n_{2}^{\prime}, n_{3}^{\prime}\right)} d \mathbf{x}
$$

Integrating the first term on the left-hand side of (A21) by parts and using the fact that $\mathbf{u}_{\left(n_{1}^{\prime}, n_{2}^{\prime}, n_{3}^{\prime}\right)}$ satisfies (A9), we have

$$
\begin{array}{r}
\int_{\Omega} \Delta \mathbf{u} \cdot \Delta \mathbf{u}_{\left(n_{1}^{\prime}, n_{2}^{\prime}, n_{3}^{\prime}\right)} d \mathbf{x}=\int_{\Omega} \mathbf{u} \cdot \Delta \Delta \mathbf{u}_{\left(n_{1}^{\prime}, n_{2}^{\prime}, n_{3}^{\prime}\right)} d \mathbf{x} \\
=\frac{16 \pi^{4}}{L^{4}}\left(n_{1}^{\prime 2}+n_{2}^{\prime 2}+n_{3}^{\prime 2}\right) \int_{\Omega} \mathbf{u} \cdot \mathbf{u}_{\left(n_{1}^{\prime}, n_{2}^{\prime}, n_{3}^{\prime}\right)} d \mathbf{x}
\end{array}
$$

so, by (A21), (A22),

$$
\left[\frac{16 \pi^{4}}{L^{4}}\left(n_{1}^{\prime 2}+n_{2}^{\prime 2}+n_{3}^{\prime 2}\right)-\lambda\right] \int_{\Omega} \mathbf{u} \cdot \mathbf{u}_{\left(n_{1}^{\prime 2}, n_{2}^{\prime 2}, n_{3}^{\prime 2}\right)} d \mathbf{x}=0 .
$$

However, using (A14) in conjunction with (A3), (A4), we get

$$
\int_{\Omega} \mathbf{u} \cdot \mathbf{u}_{\left(n_{1}^{\prime}, n_{2}^{\prime}, n_{3}^{\prime}\right)} d \mathbf{x}=\int_{\Omega}\left|\mathbf{u}_{\left(n_{1}^{\prime}, n_{2}^{\prime}, n_{3}^{\prime}\right)}\right| d \mathbf{x} \neq 0
$$

and the required result follows directly from (A24).

REMARK. The case $N=2$ may be handled in a manner similar to the case $n=3$. We consider functions of the form

$$
u_{i}=\sum_{i=1}^{4} C_{i j} f_{j}\left(x_{1}, x_{2}\right), \quad i=1,2,
$$

with

$$
\left\{\begin{array}{l}
f_{1}\left(x_{1}, x_{2}\right)=\cos \frac{2 \pi n_{1}}{L} x_{1} \cos \frac{2 \pi n_{2}}{L} x_{2} \\
f_{2}\left(x_{1}, x_{2}\right)=\cos \frac{2 \pi n_{1}}{L} x_{1} \sin \frac{2 \pi n_{2}}{L} x_{2} \\
f_{3}\left(x_{1}, x_{2}\right)=\sin \frac{2 \pi n_{1}}{L} x_{1} \cos \frac{2 \pi n_{2}}{L} x_{2} \\
f_{4}\left(x_{1}, x_{2}\right)=\sin \frac{2 \pi n_{1}}{L} x_{1} \sin \frac{2 \pi n_{2}}{L} x_{2}
\end{array}\right.
$$

The result for $n=2$ which corresponds to Lemmas A1 and A2 for $n=3$ is 
LEMmA A3. For $n=2$, the set of numbers

$$
\left\{\frac{16 \pi^{4}}{L^{4}}\left(n_{1}^{2}+n_{2}^{2}\right) \neq 0 \mid n_{1}, n_{2} \text { are nonnegative integers }\right\}
$$

contains all the eigenvalues of $\mathbf{A}$.

REMARK. Once we have shown that for nonnegative integers $n_{i}(i=1,2,3)$ the eigenvalues of $\mathbf{A}$ consist of the numbers

$$
\begin{gathered}
\lambda=\frac{16 \pi^{4}}{L^{4}}\left(n_{1}^{2}+n_{2}^{2}+n_{3}^{2}\right) \neq 0 \quad(n=3), \\
\lambda=\frac{16 \pi^{4}}{L^{4}}\left(n_{1}^{2}+n_{2}^{2}\right) \neq 0 \quad(n=2),
\end{gathered}
$$

the validity of the spectral gap condition is a consequence of standard known results on the difference of consecutive numbers which can be expressed as the sum of squares of nonnegative integers, e.g., [63]; in fact, as a consequence of such results it follows that, for the bipolar problem, condition (i) of Theorem 6.1 is satisfied, in $\operatorname{dim} n=2$, for arbitrary $\mu_{1}>0$, if $N$ is sufficiently large, but in $\operatorname{dim} n=3$ only when $\mu_{1}$ is sufficiently large.

\section{REFERENCES}

[1] A. E. Green and R. S. Rivlin, Simple force and stress multipoles, Arch. Rational Mech. Anal. 16, $325-353$ (1964)

[2] A. E. Green and R. S. Rivlin, Multipolar continuum mechanics, Arch. Rational Mech. Anal. 17, 113-147 (1964)

[3] J. L. Bleustein and A. E. Green, Dipolar fluids, Internat. J. Engrg. Sci. 5, 323-340 (1967)

[4] J. Nečas and M. Šilhavý, Multipolar viscous fluids, Quart. Appl. Math. 49, 247--265 (1991)

[5] H. Bellout, F. Bloom, and J. Nečas, Phenomenological behavior of multipolar viscous fluids, Quart. Appl. Math. 50, 559-583 (1992)

[6] M. Shinbrot, Lectures on Fluid Mechanics, Gordon and Breach, New York, 1993

[7] D. Ruelle, The turbulent fluid as a dynamical system, in New Perspectives in Turbulence, L. Sirovich, ed., Springer-Verlag, Berlin, 1991, pp. 123-138

[8] O. Ladyzhenskaya, The Mathematical Theory of Viscous Incompressible Flow, Gordon and Breach, New York, 1969

[9] O. Ladyzhenskaya, New equations for the description of viscous incompressible fluids and solvability in the large of the boundary value problems for them, Proc. Steklov Inst. Math. 102, 95-118 (1970)

[10] S. Kaniel, On the initial-value problem for an incompressible fluid with nonlinear viscosity, J. Math. Mech. 19, 681-706 (1970)

[11] Q. Du and M. D. Gunzburger, Analysis of a Ladyzhenskaya model for incompressible viscous flow, J. Math. Anal. Appl. 155, 21-45 (1991)

[12] J. L. Lions, Quelques Méthodes de Résolution des Problèmes aux Limites Non Linéaires, Dunod, Paris, 1969

[13] Y.-R. Ou and S. S. Sritharan, Analysis of regularized Navier-Stokes equations. I, Quart. Appl. Math. 49, 651-685 (1991)

[14] Y.-R. Ou and S. S. Sritharan, Analysis of regularized Navier-Stokes equations. II, Quart. Appl. Math. 49, 687-728 (1991)

[15] R. Temam, Infinite Dimensional Dynamical Systems in Mechanics and Physics, Springer-Verlag, New York, 1988

[16] R. A. Toupin, Theories of elasticity with couple stress, Arch. Rational Mech. Anal. 17, 85-112 (1964)

[17] H. Bellout, F. Bloom, and J. Nečas, Existence, uniqueness, and stability of solutions to the initial boundary value problem for bipolar viscous fluids, Differential Integral Equations 8, 453-464 (1995) 
[18] H. Bellout, F. Bloom, and J. Nečas, Young measure-valued solutions for non-Newtonian incompressible fluids, Comm. Partial Differential Equations 19, 1763-1803 (1994)

[19] H. Bellout, F. Bloom, and J. Nečas, Solutions for incompressible non-Newtonian fluids, C. R. Acad. Sci. Paris Sér. I Math. 317, 795-800 (1993)

[20] J. Nečas, Sur les normes équivalentes dans $W_{p}^{\left(k^{*}\right)}(\Omega)$ et sur la coercivité des formes formellement positives, Équations aux Dérivées Partielles, Presses Univ. Montréal, Montréal, 1965

[21] H. Bellout and F. Bloom, Existence and asymptotic stability of time-dependent Poiseuille flows of isothermal bipolar fluids, Applicable Anal. 50, 115130 (1993)

[22] H. Bellout and F. Bloom, On the uniqueness of plane Poiseuille solutions of the equations of incompressible bipolar fluids, Internat. J. Engrg. Sci. 31, 1535-1549 (1993)

[23] H. Bellout and F. Bloom, Steady plane Poiseuille flows of incompressible multipolar fluids, Internat. J. Non-Linear Mech. 23, 503518 (1993)

[24] F. Bloom and W. Hao, Steady flows of nonlinear bipolar viscous fluids between rotating cylinders, Quart. Appl. Math. 53, 143-171 (1995)

[25] H. Bellout, F. Bloom, and J. Nečas, Bounds for the dimensions of the attractors of nonlinear bipolar viscous fluids, Asymptotic Anal. 11, 131167 (1995)

[26] F. Bloom, Attractors of bipolar and non-Newtonian viscous fluids, First World Congr. Nonlinear Analysts (Tampa, FL, August 1992), Vol. I, V. Lakshmikantham, ed., Walter de Gruyter Publishers, 1996, pp. 583-596

[27] F. Bloom, Attractors of non-Newtonian fluids, J. Dynamics and Differential Equations 7, 109-140 (1995)

[28] P. Constantin, C. Foias, and R. Temam, Attractors Representing Turbulent Flows, Mem. Amer. Math. Soc. No. 314 (1985)

[29] P. Constantin, C. Foias, and R. Temam, On the dimension of the attractors in two-dimensional turbulence, Phys. D. 30. 284296 (1988)

[30] J. Malek and J. Nečas, A finite-dimensional attractor for thrę-dimensional flow of incompressible fluids, J. Differential Equations, in press

[31] C. Foiaş and G. R. Sell, Inertial manifolds for nonlinear evolutionary equations, J. Differential Equations 73, 309-353 (1988)

[32] P. Constantin, C. Foiaş, B. Nicolaenko, and R. Temam, Integral Manifolds and Inertial Manifolds for Dissipative Partial Differential Equations, Springer-Verlag, New York. 1989

[33] P. Constantin and C. Foiaş, Navier-Stoker Equations, University of Chicago Press, Chicago, 1988

[34] M. W. Smiley, Global attractors and approximate inertial manifolds for nonautonomous dissipative equations, Applicable Anal. 50, 217241 (1993)

[35] G. R. Sell and Y. You, Inertial manifolds : the nonselfadjoint case. J. Differential Equations 96. 203-255 (1992)

[36] M. Kwak, Finite-dimensional inertial forms for the $2 D$ Navier-Stokes equations, Indiana Univ. Math. J. 41, 927-981 (1993)

[37] G. R. Sell and J. Mallet-Paret. Inertial manifolds for reaction diffusion equations in higher space dimensions, J. Amer. Nath. Soc. 1, 805-866 (1988)

[38] M. Luskin and G. R. Sell, Parabolic regularization and inertial manifolds, IMA Preprint. University of Minnesota, Minneapolis, 1989

[39] P. Constantin, C. Foiaş, B. Nicolaenko, and R. Temam, Spectral barriers and inertial manifolds for dissipative partial differential equations, J. Dynamics and Differential Equations 1, 45-73 (1989)

[40] C. Foias,, G. R. Sell, and E. Titi, Exponential tracking and approximation of inertial manifolds for dissipative nonlinear equations, J. Dynamics and Differential Equations 1, 199-244 (1989)

[41] M. Marion, Approximate inertial manifolds for reaction-diffusion equations in higher space dimensions, J. Dynamics and Differential Equations 1, 245- 267 (1989)

[42] M. Luskin and G. R. Sell, The construction of inertial manifolds for reaction-diffusion equations by elliptic regularization, IMA Preprint, University of Minnesota, Minneapolis, 1989

[43] E. Titi, On approximate inertial manifolds to the Navier-Stokes equations, J. Math. Anal. Appl. $149,540-557(1990)$

[44] P. Constantin, A construction of inertial manifolds, in The Connection Between Infinite-Dimensional and Finite-Dimensional Dynamical Systems (Boulder, CO, 1987), Contemp. Math., vol. 99, Amer. Math. Soc., Providence, RI, 1989, pp. 2762

[45] F. Demenge and J. M. Ghidaglia, Some remarks on the smoothness of inertial manifolds. Nonlinear Anal. 16, $79 \cdot 87(1991)$ 
[46] A. Eden, C. Foiaş, B. Nicolaenko, and R. Temam, Inertial sets for dissipative evolution equations, IMA Preprint, University of Minnesota, Minneapolis, 1990; cf. Ensembles inertiels pour des équations d'évolution dissipatives, C. R. Acad. Sci. Paris Sér. I Math. 310, 559-562 (1990)

[47] M. Marion, Inertial manifolds associated to partly dissipative reaction-diffusion systems, J. Math. Anal. Appl. 143, 295-326 (1989)

[48] R. Temam, Induced trajectories and approximate inertial manifolds, RAIRO Modél. Math. Anal. Numér. 23, 541-561 (1989)

[49] J. Heywood and R. Rannacher, On the question of turbulence modelling by approximate inertial manifolds and the nonlinear Galerkin method, SIAM J. Numer. Anal. 30, 1603 1621 (1993)

[50] J. K. Hale, Asymptotic Behavior of Dissipative Systems, Amer. Math. Soc., Providence, RI, 1988

[51] A. V. Babin and M. I. Vishik, Attractors of Evolution Equations, North-Holland, Amsterdam, 1992

[52] A. V. Babin and M. I. Vishik, Attractors of partial differential evolution equations and estimates of their dimension, Russian Math. Surveys 38, no. 4, 151-213 (1983)

[53] A. V. Babin and M. I. Vishik, Attractors for the Navier-Stokes system and for parabolic equations and estimates of their dimension, J. Soviet Math. 28, 619-627 (1983)

[54] O. A. Ladyzhenskaya, A dynamical system generated by the Navier-Stokes equations, J. Soviet Math. 3, 458-479 (1975)

[55] C. Foiaş and R. Temam, The connection between the Navier-Stokes equations, dynamical systems, and turbulence theory, in Directions in Partial Differential Equations, Academic Press, NY, 1987, pp. $55-73$

[56] A. V. Babin and M. I. Vishik, Regular attractors of semigroups and evolution equations, J. Math. Pures Appl. 62, 441-491 (1983)

[57] A. V. Babin and M. I. Vishik, Attractors of partial differential evolution equations in an unbounded domain, Proc. Roy. Soc. Edinburgh Sect. A 116, 221-243 (1990)

[58] A. V. Babin, The attractor of a Navier-Stokes system in an unbounded channel-like domain, J. Dynamics and Differential Equations 4, 555-584 (1992)

[59] P. Constantin, C. Foiaş, O. Manky, and R. Temam, Determining modes and fractal dimension of turbulent flows, J. Fluid Mech. 150, 427-440 (1985)

[60] F. Abergel, Attractor for a Navier-Stokes flow in an unbounded domain, RAIRO Modél. Math. Anal. Numér. 23, 359-370 (1989)

[61] F. Abergel, Existence and finite dimensionality of the global attractor for evolution equations on unbounded domains, J. Differential Equations 83, 85 108 (1990)

[62] J. M. Ghidaglia and R. Temam, Attractors for damped nonlinear hyperbolic equations, J. Math. Pures et Appl. 66, 273-319 (1987)

[63] Ian Richards, On the gap between numbers which are the sum of two squares, Adv. Math. 46, 1-2 (1982) 